\title{
Limits to Ductility Set by Plastic Flow Localization
}

by A. Needleman and J. R. Rice

\author{
Division of Engineering \\ Brown University \\ Providence, R.I. 02912
}

Paper presented at General Motors Research Laboratories Symposium on Mechanics of Sheet Metal Forming: Material Behavior and Deformation Analysis, 17-18 October 1977, Warren, Michigan.

To be published in Symposium Proceedings, Plenum Press, New York, 1978. 


\section{DISCLAIMER}

This report was prepared as an account of work sponsored by an agency of the United States Government. Neither the United States Government nor any agency Thereof, nor any of their employees, makes any warranty, express or implied, or assumes any legal liability or responsibility for the accuracy, completeness, or usefulness of any information, apparatus, product, or process disclosed, or represents that its use would not infringe privately owned rights. Reference herein to any specific commercial product, process, or service by trade name, trademark, manufacturer, or otherwise does not necessarily constitute or imply its endorsement, recommendation, or favoring by the United States Government or any agency thereof. The views and opinions of authors expressed herein do not necessarily state or reflect those of the United States Government or any agency thereof. 


\section{DISCLAIMER}

Portions of this document may be illegible in electronic image products. Images are produced from the best available original document. 


\section{Abstract}

The theory of strain localization is reviewed with reference both to local necking in sheet metal forming processes and to more general three dimensional shear band localizations that sometimes mark the onset of ductile rupture. Both bifurcation behavior and the growth of initial imperfections are considered. In addition to analyses based on classical Mises-like constitutive laws, we discuss approaches to localization based on constitutive models that may more accurately model processes of slip and progressive rupturing on the microscale in structural alloys. Among these non-classical constitutive features are the destabilizing roles of yield surface vertices and of non-normality effects, arising, for example, from slight pressure sensitivity of yield. We also discuss analyses based on a constitutive model of a progressively cavitating dilational plastic material which is intended to model the process of ductile void growth in metals. A variety of numerical results are presented. In the context of the three dimensional theory of localization, we show that a simple vertex model predicts ratios of ductility in plane strain tension to ductility in axisymmetric tension qualitatively consistant with experiment. We also illustrate the destabilizing influence of a hydrostatic stress dependent void nucleation criterion. In the sheet necking context, and focussing on positive biaxial stretching, it is shown that forming limit curves based on a simple vertex model and those based on a simple void growth model are qualitatively in accord, although attributing instability to very different physical mechanisms. These forming limit curves are compared with those obtained from the Mises material model and employing various material and geometric imperfections. 


\section{Introduction}

A remarkably common observation in highly deformed ductile solids is that a smooth deformation pattern rather abruptly gives way to one involving a band (or bands) of localized deformation. Here, ve review a theoretical framevork which associates this localization of deformation with a material instability. Hore specifically, we consider an element of a solid, constrained so as to rule out geometric instabilities, subject to loading that could give rise to a homogeneous deformation and determine the conditions under which the constitutive relation of the solid permits a highly localized deformation pattern to emerge. Within this framework, the onset of localization can occur as a bifurcation from a homogeneous deformation state or it can be triggered by some initial inhomogeneity, possibly well hefore a bifurcation would occur if the (small) inhomogeneity were absent. Although not all observed localization phenomena can be expected to fit vithin this theoretical framework, it is broad enough to encompass descriptions of the localized necking mode characteristic of ductile metal sheets (when these are viewed as two dimensional continua) as well as of the "shear band" localizations that seem to be important precursors to ductile rupture.

As is not surprising, given the point of view adopted here, the onset of localization will depend critically on the assumed constitutive law. We base our analyses on the classical rate independent Prandtl-Reuss equations (suitably modified to account for finite deformations) and departures from this idealized model that include the effects of (i) yield surface vertices, (ii) deviations from plastic "normality" and (iii) the dilational plastic flow (and possiple strain softening) due to the nucleation and growth of voids.

The cffects tof these deviations from the idealized Prandtl-Reuss material 
model on the predicted onset of flow locaiization are illustrated and compared. Particular emphasis is given to the example of local necking in metal sheets subject to positive biaxial stretching, where both vertex effects and the weakening induced by ductile void growth appear to provide plausible explanations of observed behavior.

\section{Constitutive Relations}

The constitutive relations on which we base our discussion are the classical Prandtl-Reuss relations and generalizations of these to allow investigation of (i) yield surface vertex effects as predicted, e.g., from models of the crystalline slip process, (ii) slight pressure-sensitivity of yield and deviations from an associated flow rule, and (iii) dilational plastic flow, and, possibly, strain softening due to micro-rupture by the nucleation and growth of cavities. Nlthough not considered explicitly here, significant departures from isotropy are also important in applications to sheet materials.

We wite the rate of deformation $D$ (symmetric part of $\partial v / \partial y$, where $\checkmark$ is the particle velocity) as.

$$
D=D^{e}+D^{P}
$$

where, assuming elastic isotropy and small elastic dimension changes, the elastic part, $D^{e}$, is

$$
D^{e}=\frac{I}{2 G}\left[\stackrel{\nabla}{\sigma}-\frac{\nu}{I+\nu} \underset{\sim}{I} \operatorname{tr}(\stackrel{\nabla}{\sigma})\right]
$$

Here $\operatorname{tr}()$ denotes the trace, $\underset{\sigma}{\sigma}$ is the Jaumann (or corotational) derivative of Cauchy (true) stress and is related to the ordinary material time rate $\dot{\sigma}$ by 


$$
-\stackrel{\nabla}{\sigma}=\dot{\sigma}+\sigma \cdot \Omega-\Omega \cdot \sigma
$$

where the spin $\Omega$ is the anti-symmetric part of $\partial v / \partial x$. The plastic part, $D^{0}$, according to the Prandtl-Reuss model, is (e.g., Hill [1])

$$
D_{i j}^{p}=\frac{i}{2 h} \frac{\sigma_{i j}}{\tau_{e}} \dot{\tau}_{e}=\frac{1}{4 h} \frac{\sigma_{i j}^{\prime} \sigma_{k 2}^{\prime}}{\tau_{e}^{2}}{\stackrel{\sigma}{\sigma_{k 2}}}^{\sigma_{2}}
$$

where $\sigma^{\prime}$ is the deviatoric part of $\sigma, h$ is the plastic hardening modulus in shear and $\tau_{e}$ is the "equivalent" shear stress, related to the equivalent tensile stress $\sigma_{e}$ and to $\sigma$ by

$$
\tau_{e}^{2}=\frac{1}{3} \sigma_{e}^{2}=\frac{1}{2} \sigma_{i j}^{\prime} \sigma_{i j}^{\prime}
$$

of course, the above form for $\mathrm{D}^{\mathrm{P}}$ is understood to apply only when the stress state is at yield and the imposed deformations are such as to enforce continued plastic flow; otherwise ${ }_{\sim}^{P}=\underset{\sim}{0}$. The value of the equivalent shear stress $\tau_{e}$ required for continued yielding, is considered to be a function of the equivalent "engineering" plastic shear strain $\gamma_{e}^{p}$, or, alternatively, $\sigma_{e}$ is taken to be a function of the tensile equivalent plastic strain $\varepsilon_{e}^{p}$, where

$$
\dot{\gamma}_{e}^{p}=\sqrt{3} \dot{\varepsilon}_{e}^{p}=\left(2 D_{i j}^{P_{i j}^{\prime}} D_{i j}^{P^{\prime}}\right)^{1 / 2},
$$

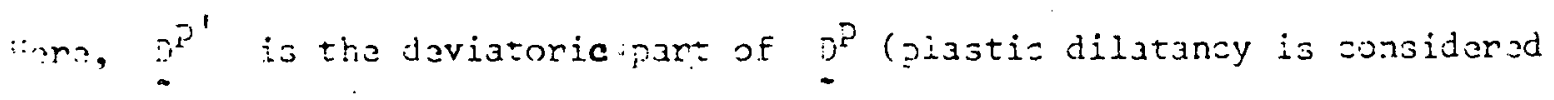
subsequently) and the plastic hardening modulus is given by

$$
h=d \tau_{e} / d \gamma_{e}^{p}=\frac{1}{3} d \sigma_{e} / d \varepsilon_{e}^{p}
$$

as depicted in Fig. 1.

We remark that these constitutive relations involve a $\mathrm{D}^{\mathrm{P}}$ that has a direction "nomal" to the yield surface in stress space. Furthermore, this surfice is smooth, with a unique normal at the current state. Therefore, the

$$
\therefore\left[\sigma_{\sim}^{\sigma}\right]_{i j}=\sigma_{i k} \Omega_{k j}
$$


direction of $D^{P}$ is independent of that of the stress rate $\stackrel{\nabla}{\sigma}$ and, in fact, the yield surface is imagined to expand isotropically, having the mathematical expression $\tau_{e}=f\left(\gamma_{e}^{P}\right)$. The criterion for yield is pressure insensitive and the plastic flow is volume preserving (the two go together when normality is assumed ).

Now, investigations of the stability of plastic flow against localization '(Rudnicki and Rice [2]; Rice [3]) suggest that results can be very sensitive to deviations from the various attributes just discussed. Thus we consider several generalizations of the Prandtl-Reuss relations and, following a formulation of conditions for localization in the next section, we examine their consequences.

First consider the smooth yield surface assumption, with its consequent requirement that the direction of $\mathrm{D}^{\mathrm{P}}$ is not influenced by that of $\underset{\sigma}{\nabla}$. This assumption is at variance with models for polycrystaline aggregates based on single crystal plasticity. Hill [4] remarks that the associated discreteness of slip systems in each grain leads to the prediction of a vertex on the yield surface, at least when the yield surface is defined for very small offset plastic strains. Specific calculations for various polycrystal models carried out by Lin [5] and Hutchinson [6] do, in fact, exhibit such yield surface vertices. Unfortunately, no simple mathematical formulation of response at a vertex has yet been developed, but an approximate model, useful when the prior deformation has heen carried out at conditions near to proportional stressing, has been developed by Rudnicki and Rice [2] and Stören and Rice [7]. These authors introduce two hardening moduli; $h$, as introduced before, characterizes the hardening when $\stackrel{\nabla}{\sigma}$ ' merely continues the direction of $g^{\prime}$. But owing to vertex effects, a new modulus $?_{1}(>h)$ 
comes into play to characterize the response to that portion of $\underset{\sigma}{\sim}$ directed tangentially to what is taken as the yield surface in the isotropic hardening model. This is illustrated schematically in Fig. 2. The adopted form is

$$
D_{i j}^{P}=\frac{l}{2 h} \sigma_{i j}^{\prime} \frac{\dot{\tau}_{e}}{\tau_{e}}+\frac{l}{2 h_{1}}\left(\stackrel{r}{\sigma}_{i j}-v_{i j} \frac{\dot{\tau}_{e}}{\tau_{e}}\right)
$$

where it is assumed that $\underset{\sigma}{\nabla^{\prime}}$ is not strongly different in direction from $\sigma^{\prime}$. In the related area of the plastic buckling of structures, it is well known that the simple $\mathrm{J}_{2}$ "deformation" theory of plasticity gives bifurcation loads more in accord with experimental buckling loads than the corresponding bifurcation loads of $\mathrm{J}_{2}$ flow theory, when the predictions of these two theories differ. Despite initial objections, the use of deformation theory in . bifurcation analyses was justified, as originally noted by Batdorf [45], in terms of the presence of a yield surface vertex at the current state. For a recent discussion of this issue, see [46]. The slip theory of Batdorf and Budiansky [47] did give rise to such a vertex and to path-independent stressstrain relations, i.e., deformation theory, for all loading paths which monotonically stressed all active slip systems. However, this deformation theory involves both $\mathrm{J}_{2}$ and the third invariant. Subsequently, Sanders [8] constructed a flow theory based on infinitely many linear loading functions that for stressing paths that were "fully active," in the sense that every loading function, once stressed to yield, does not unload, coincided with simple $\mathrm{J}_{2}$ "deformation" theory. Sanders' considerations were based on infinitesimal strain and several generalizations of deformation theory to large strains were examined by Storren and Rice. In particular, they showed that if a deformation measure of the type

$$
\int D^{p} d t
$$


but suitably modified to eliminate effects of the spin history $?$ on reorientation of material directions, is taken to be coaxial with $\sigma$ ' (again, corrected for rotations), with the ratio between the two depending on $\tau_{e}$, then the expression $(2.8)$ for $D^{P}$ results with

$$
h_{1}=\tau_{e} / \gamma_{e}^{p}=\frac{1}{3} \sigma_{e} / \varepsilon_{e}^{p} .
$$

That is, the vertex modulus $h_{1}$ is the plastic "secant modulus" as illustrated in Fig. 1. Storren and Rice mention other possible generalizations of deformation theory and one of these, based on the logarithmic strain tensor, is adopted by Hutchinson and Neale [9] in a companion paper in this volume. As discussed in [23] and [9] the value assumed by the vertex modulus $h_{1}$ can, in certain circumstances, depend significantly on the choice of deformation measure on which the deformation theory is hased.

Although a deformation theory type formulation of yield vertex effects may be appropriate for the analysis of bifurcations from previously homogeneous, and nearly proportional, deformation paths such a model is undoubtedly not versatile enough to treat finite stress alterations in a direction different from that before bifurcation.

As a means of investigating the effects of dilatant plasticity and pressure sensitive yielding, in a manner that includes the possibility that these do not satisfy an associated flow rule (i.e., "non-nomality"), we adont the simple generalization of the Prandtl-Reuss equations studied by Rudnicki and Rice [2]. In this case we write

$$
D_{i j}^{P}=\frac{1}{h} P_{i j} Q_{k l} \stackrel{\nabla}{\sigma_{k l}}
$$

where

$$
P_{i j}=\frac{\sigma_{i j}^{\prime}}{2 \tau_{e}}+\frac{\beta}{3} \delta_{i j}, \quad Q_{k \ell}=\frac{\sigma_{k \ell}^{\prime}}{2 \tau_{e}}+\frac{\mu}{3} \delta_{k \ell} .
$$

Hara, p gives the "direction" of plastic flow (and in this sense, vertex affects are not included since $?$ is assumed independent of $\stackrel{\nabla}{\sim}$ ) while? 
gives the outer "normal" to the yield surface in stress space. When ' $\beta$ and $\mu$ vanish this reduces to the Prandtl-Reuss form (2.4). Wore generally, $B$ gives the ratio of plastic dilatancy to equivalent plastic shear,

$$
D_{r k}^{p}=3 \dot{\gamma}_{e}^{p}
$$

wheras $\mu$ measures the pressure sensitivity of yield (as sketched in Fig. 3) in that the amount of a plastic deformation increment depends on

$$
d \tau_{e}+\mu d\left(\sigma_{k k} / 3\right)
$$

Note that plastic "normality" applies only when $\mu=B \quad$ (i.e., shen $P=Q$ ). Rudnicki and Rice motivate such a constitutive model. with reference to the dilatant, frictional response of rocks. However, there are specific cases in metal plasticity where the same representation seems apnlicable. First, Spitzig et al. [10] report strength differential (SD) effects in martensitic high strength steels, whereby the compressive yield strength is finitely different than that for tension. Spitzig et al. present evidence that the SD arises from the effect of the mean stress (rather than, say, the third stress invariant) on the value of ' $\mathrm{t}$ e required for yield, and hence the effect would seem to fit into the framework of the present formulation. Since the SD is defined as

$$
S D=\frac{\sigma_{c}-\sigma_{t}}{\left(\sigma_{c}+\sigma_{t}\right) / 2}
$$

where $\sigma_{c}$ and $\sigma_{t}$ are the yịield strengths in compression and tension, respectively, the parameter $\mu$ is

$$
\mu=\frac{\sqrt{3}}{2}(S D),
$$

at least if $\mu$ is assumed to be essentially independent of the lcvel of $\sigma_{k k}$ that acts. It might, instead, increase rapidly with large values of $\sigma_{k k}$ and in, such a case (2.15) will underestimate $\mu$. Spitzig et al. find SD values in 
the range 0.05 to 0.07 for AISI 4310 and 4330 steels (although the SD value is approximately half for the unaged material and of the order 0.01 to 0.02 for HY-80 [11]; the value would seem to be much lower, near zero, for mild steels). Spitzig et al. also find that the plastic dilatancy is much smaller than required for normality, and according to their experiments, the dilatancy factor $\beta$ introduced above would have a value on the order of $\mu / 15$ and can be taken as zero.

The dilatant and pressure-sensitive plastic flow model described above arises also as a representation of the flow rule for void-containing ductile solids, at least in the approximate development of constitutive relations for this case by Gurson $[12,13]$. Based on the approximation of a solid with volume fraction $f$ of voids by a homogeneous spherical body with a concentric spherical cavity, and employing certain other approximations in the rigidplastic limit analysis of this body, Gurson suggests the combined stress yield condition

$$
\Phi(\underset{\sim}{\sigma}, \bar{\sigma}, f) \equiv \frac{3}{2} \frac{\sigma_{i j}^{\prime} \sigma_{j . j}^{\prime}}{\bar{\sigma}^{2}}+2 f \cosh \left(\frac{\sigma_{k k}}{2 \bar{\sigma}}\right)-1-f^{2}=0
$$

where $\sigma_{i j}$ is the macroscopic stress state acting on the voided material and $\bar{\sigma}$ is the tensile flow strength of the matrix material, taken as spatially uniform in the analysis. llote that the yield condition resembles the Mises form (which is assumed in Gurson's model to hold for the matrix material) but that whenever the void volume fraction is non-zero, there is an effect of the mean normal stress on plastic flow as illustrated in Fig. 4. Indeed, McClintock [14] has noted the exponential amplification of hole growth rates over remotely applied plastic strain rates at high values of $\sigma_{k k} / \bar{\sigma}$. !low, as noted hy Horg. [16], an argument of Bishop and Hill [15] is relevant in this context and, since each element of matrix material is assumed to satisfy 
the normality rule basect on the Mises yield condition, it requires the macro-

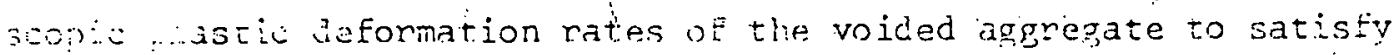

$$
D_{i j}^{P}=\Lambda \frac{\partial P}{\partial \sigma_{i j}}
$$

where $\Lambda$ is to be determined in a manner consistent with the strain hardening behavion of the material. Following Gurson, we assume that the yield function remains effectively isotropic (e.g., deviations of the void shape from spherical are $n e g l$ lected), in the sense that (2.16) continues to describe the yield function and that only the matrix flow strength $\bar{\sigma}$ and void volume fraction $f$ vary during the deformation. The matrix equivalent plastic tensile strain $\bar{\varepsilon}^{\mathrm{P}}$ is assumed to vary according to the equivalent plastic work expression

$$
(1-f) \bar{\sigma} \bar{\varepsilon} p=\sigma_{i j} D_{i j}^{p}
$$

so that

$$
\dot{\bar{\sigma}}=\bar{h} \frac{\sigma_{i j}}{(l-f) \bar{\sigma}} D_{i j}^{p}
$$

where $\bar{h}=\mathrm{d} \vec{\sigma} / \mathrm{d} \bar{\varepsilon}^{\mathrm{p}}$ is the equivalent tensile hardening rate of the matrix material (note that $h$ as introduced earlier represented an equivalent shear hardening rate). The void volume fraction increases because of the growth of existing voids, which causes $\dot{f}$ to increase at the rate $(1-f) D_{k k}^{P}$, and because of the nucleation of new voids, say by the cracking or decohesion of inclusions or second-phase particles. Accordingly we write

$$
\begin{aligned}
\dot{f} & =(\dot{f})_{\text {growth }}+(\dot{\mathrm{f}})_{\text {nucleation }} \\
& =(1-f) \mathrm{D}_{k k}^{\mathrm{p}}+A \dot{\bar{\sigma}}+B \dot{\sigma}_{k k} / 3 .
\end{aligned}
$$

Here the nucleation rate has been represented in terms of the two parameters $A$ and $B$. Note that if the nucleation of cavities can he correlated exclusively in terms of the equivalent plastic strain (or, since they are related, in terms of $\bar{\sigma})$, as suggested by Gurson's $[12,17]$ analysis of data 
by Gurland [19] on a spheroidized carhon steel, then $B=0$. On the other hand, if the nucleation criterion depends only on the maximum stress transmitted across the particle-matrix interface, as suggested in studies by Argon et al. [19-21], then $B \approx A$ (assuming that $\bar{\sigma}+\sigma_{k k} / 3$ is an approximation to this maximum stress). More elaborate nucleation models, based on an extension of Argon et al.'s [21] considerations of the statistics of particle spacing, are discussed by Gurson $[12,17]$.

Now, since it is assumed that $\phi(\underset{\sim}{\sigma}, \vec{\sigma}, f)=0$ throughout a program of sustained plastic deformation, the "consistency", condition $\dot{\phi}=0$ follows:

$$
\begin{aligned}
0 & =\frac{\partial \Phi}{\partial \sigma_{k \ell}} \dot{\sigma}_{k \ell}+\frac{\partial \phi}{\partial f} \dot{f}+\frac{\partial \Phi}{\partial \bar{\sigma}} \dot{\sigma} \\
& =\frac{\partial \Phi}{\partial \sigma_{k \ell}} \stackrel{\nabla}{\sigma \ell}_{k l}+\frac{\partial \Phi}{\partial f} \frac{B}{3} \stackrel{\nabla}{\sigma k}_{k} \\
& +\left[\frac{\partial \phi}{\partial f}(1-f) \delta_{i j}+\left(\frac{\partial \phi}{\partial f} A+\frac{\partial \Phi}{\partial \bar{\sigma}}\right) \overline{\mathrm{h}} \frac{\sigma_{i j}}{(1-f) \bar{\sigma}}\right] \wedge \frac{\partial \phi}{\partial \sigma_{i j}}
\end{aligned}
$$

where, in the last version, we have used $(2.18,19)$ for $\dot{\bar{\sigma}}, \dot{\mathrm{f}}$ and also used (2.17) for $D_{i j}^{\mathrm{P}}$. From this result we can solve for $\Lambda$ and the resulting plastic flow rule has the form

$$
D_{i j}^{D}=\frac{1}{H} \frac{\partial \Phi}{\partial \sigma_{i j}}\left(\frac{\partial \phi}{\partial \sigma_{k \ell}}+\frac{\partial \phi}{\partial E} \quad \frac{B}{3} \delta_{k \ell}\right) \nabla_{k \ell}
$$

where

$$
H=-\left[\frac{\partial \phi}{\partial f}(1-f) \delta_{i j}+\left(\frac{\partial \Phi}{\partial f} A+\frac{\partial \Phi}{\partial \bar{\sigma}}\right) \bar{h} \frac{\sigma_{j . j}}{(1-f) \bar{\sigma}}\right] \frac{\partial \phi}{\partial \sigma_{i j}} .
$$

In fact, given the form of $\phi$ in $(2.16)$, this plastic flow rule is identical to that introduced in eqs. $(2.10,11)$ provided that the parameters $\therefore, 3$ and, 1 are identified as 


$$
\begin{gathered}
h=\frac{\dot{\bar{h}}}{3} \frac{\left(1+\dot{f}^{2}-2 f c+f T s\right)^{2}}{(1-f)\left(1+f^{2}-2 f c\right)} \\
-\frac{(c-f) \bar{\sigma}}{6\left(1+\overline{\mathrm{F}}^{2}-2 f c\right)}\left[3 f(1-f) s+2 A \bar{h} \frac{1+f^{2}-2 f c+f \Sigma s}{1-f}\right], \\
B=\frac{\sqrt{3} \dot{I} s}{2 \sqrt{1+f^{2}-2 f c}}, \\
\mu=8+\frac{(c-f) B \bar{\sigma}}{\sqrt{3} \sqrt{1+f^{2}-2 f c}},
\end{gathered}
$$

and where we have used the shortened notations

$$
\Sigma \equiv \sigma_{k k} / 2 \bar{\sigma}, s \equiv \sinh \Sigma, c \equiv \cosh \Sigma
$$

We note that $\mu$ differs from $B$, so that normality does not apply, whenever $B \neq 0$. That is, for a maximum-stress-dependent criterion for cavity nucleation (so that $B \neq 0$ ) the plastic flow direction $P$ and effective normal $\underset{\sim}{0}$ to the yield surface are not coincident. The point is discussed at some length by Gurson $[12,17]$, who observes that there is no unique direction $Q$ when $B \neq 0$. The direction given by (2.11) and the above. $\mu$ applies if the stress increments acting serve to continue the void nucleation process. If the stress increments do not cause further nucleation, $B$ can be replaced by zero and then $\mu$ and $O$ agree with $\beta$ and $P$ so that "normality" appljes.

It may be noted further that the macrosconic hardening rate $h$ in (2.22) can be negative. This is promoted, particularly, by large triaxial tension so that $c$ and $s$ are large. For example, assume for simplicity. that the void nucleation parameter $A$ is zero, that $f$ and cven $f$ cosh $\Sigma$ are small compared to unity, but that the mean normal stress is high so that $\cosh \Sigma \approx \sinh \Sigma \approx \mathrm{e}^{\Sigma / 2}$. Then

$$
h \approx \bar{h}-f \frac{\bar{\sigma}}{\bar{z}} \exp \left(\frac{\bar{\sigma} k}{\bar{\sigma}}\right) \text {, }
$$


and if $\bar{h}=\bar{\sigma}$ (i.e., the matrix is deformed to a strain equal to that for tensile necking) and $\sigma_{k k}=\sqrt{3}(1+\pi) \bar{\sigma}$, as appropriate to the Prandt stress trizxiality ahead of a sharp crack in plane strain [22], then $h$ will be negative whenever $\tilde{f}>0.002$. Of course, when $A>0$ the softening is yet more pronounced.

Applications of the Gurson constitutive model to localization will be made subsequently. However, it is important to realize that the model involves several rather arbitrary approximations and assumptions, rot yet clearly tested. against more rigorous models or against experiment. Hence the predictions based on it should be regarded as suggestive at best. 


\section{Analysis of Localization}

The point of view taken here regards the localization of defomation as an instability that can be predicted in terms of the pre-localization constitutive relation of the material. The basic principles were given by Hadamard [25] for elastic solids and extended to elastic-plastic solids by Hill [26], Thomas [44], and Mandel [27]. Not all localization ohenomena can be expected to fit within this Eramework and limitations inherent to this approach have been discussed by Rice [3].

We model the material as rate indesendent and seek conditions under: which the deformations concentrate into a narrow band. Within this framework localization can take place when the constitutive relation allows a bifurcation from a homogeneous deformation field into a highly concentrated band mode. Altennatively, it may be triggered by some initially mall nonuniformity of material properties, possibly vell before a bifuncation would occur if the initial (small) nonuniformity were absent. Here, Darticular emohasis is given to Rice's [3] analysis of flow localization in the presence of initial inhomogeneities, which proceeds in the spirit of the MarciniakKuczynski [24] approach to localized recking in thin sheets.

We denote the position of a material point in some reference configuration by $x$ and its position in the current configuration by $\bar{x}$, where both $\underset{\sim}{x}$ and $\underset{\dot{x}}{\bar{x}}$ are referred to a fixed Cartesian frame. The deformation gradient tensor, $F$, is given by

$$
F=\frac{\partial \bar{x}}{\partial \underline{x}} \quad F_{i j}=\frac{\partial \bar{x}_{i}}{\partial x_{j}} \equiv \bar{x}_{i, j}
$$

where $F_{i j}$ are the components of the deformation gradient tensor on the fixed Cartesian axes and ()$, i$ here, and subsequently, denotes $\partial() / \partial x_{i}$. Stress is measured by the nonsymmetric nominal stress tensor $\mathfrak{s}$, 
defined so that $n, s$ is the force acting, per unit area in the reference configuration, on an element of surface having (unit) normal $\underline{n}$. It: satisfies

$$
s_{i j, i}=0
$$

at equilibrium, with body forces neglected. The nominal stress, $s$, and the true (or Cauchy) stress are related through

$$
\sigma=J^{-1} \underset{\sim}{F} \cdot s
$$

where $J$, the Jacobian, is given by

$$
J=\operatorname{det}(F)
$$

and represents the ratio of the volume of a material element in the current configuration to its volume in the reference configuration.

Now consider the solid subjected quasi-statically to an increment of deformation which in a homogenous (and homogeneously deformed) solid would give rise to the uniform field $\dot{\mathrm{F}}_{0}$. Suppose, however, that within a thin planar band of orientation $\underline{\sim}$ in the reference configuration (see Fig. 5). incremental field quantities are permitted to take on values differing from their uniform values outside this band. The band is presumed sufficiently narrow to be regarded as homogeneously deformed.

At the considered instant, the current values of field quantities and material properties inside the band may be the same as those of corresponding quantities outside the band, as in a bifurcation analysis. On the other hand, if an initial imperfection was present, the current values of field quantities and material properties inside the band will, in general, differ from those outside the band. In either case two conditions must be 
satisfied.

First, compatability requires the displacement increments to be continuous across the band. Thus (see, for example, $[3,25,26]$ ),

$$
\dot{\mathrm{F}}_{\mathrm{b}}=\underset{\sim}{\dot{\mathrm{F}}}+\underset{\sim}{\dot{q} \mathrm{n}} \text { or } \quad \Delta \dot{\vec{F}}=\dot{\sim} \boldsymbol{\sim}
$$

where ()$_{b}$ denotes field quantities within the band, ()$_{0}$ denotes corresponding quantities outside the band, $\Delta() \equiv()_{b}-()_{0}$ and (') denotes the material derivative.

The second requirement that must be met is that of incremental equilibrium. This takes the form

$$
\underline{n} \cdot \dot{s}_{\dot{\sim}}=\underset{\sim}{n} \cdot \dot{\sim}_{i}^{0} \quad \text { or } \quad n \cdot \Delta \dot{s}=0
$$

To proceed further, we assume that the relation between $\dot{s}$ and $\dot{\vec{F}}$ is piecewise linear so that

$$
\dot{s}=\underline{\sim}: \dot{F}
$$

where the tensor of moduli $\underline{K}$ is homogeneous of degree zero in $\dot{\vec{F}}$ (for rate independence) but may have a number of branches; the active branch depending on $\dot{F}$. Combining (3.5), (3.6) and (3.7) gives

$$
\left(\underline{n} \cdot k_{b} \cdot \underline{n}\right) \cdot \dot{q}=\underline{\sim} \cdot\left(K_{0}-K_{b}\right) \cdot \dot{\vec{r}}_{-0}
$$

where $K_{b}$ and $K_{-0}$ represent the active branches of the tensor of moduli inside and outside the band, respectively. Of course, both $\dot{\mathrm{F}}_{\mathrm{b}}$, which depends on $\dot{q}_{\sim}$, and $\dot{F}_{\sim}$ must be consistent with the corresponding branches of $K$.

Localization takes place when

$$
\operatorname{det}\left(n \cdot K_{b} \cdot n\right)=0
$$


Subject to the initial conditions, which specify the imperfection (if any), (3.8) constitutes a system of three equations for the three components of q which can be solved in an incremental fashion up to the point at which the localization condition (3.9) is satisfied. At any stage of the loading history the accumulated deformation in the band is simply

$$
\mathrm{F}_{\mathrm{b}}=\underset{\sim}{\mathrm{F}_{0}}+\underset{\sim}{\mathrm{qn}}
$$

An alternative formulation, phrased in terms of the Eulerian velocity gradient, $\underset{\sim}{\Gamma}=\dot{\vec{F}} \cdot \vec{F}^{-1}$, and the Cauchy (or true) stress tensor, $\underset{\sim}{\sigma}$, is sometimes convenient. From (3.5) and (3.10), it can be shown that

$$
\underset{\sim}{\Gamma_{\mathrm{b}}}=\Gamma_{0}+\underset{\sim \sim}{\dot{\mathrm{Q}} \bar{n}} \quad \text { or } \quad \Delta \Gamma=\dot{\mathrm{Q}}_{\sim} \overline{\mathrm{n}}
$$

where $\bar{n}$ is the unit normal defining the orientation of the band in the current configuration as sketched in Fig. 5 and is given by

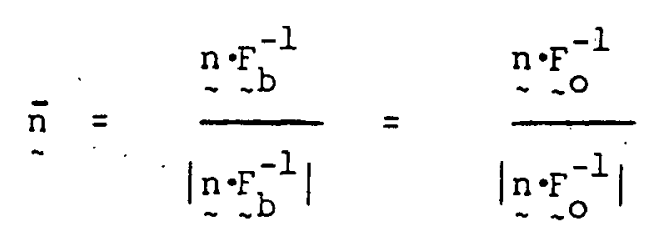

Whenever (3.5) holds incremental equilibrium is alternatively expressed by

$$
\bar{n} \cdot \Delta \dot{\sigma}+\underset{\sim}{\tilde{n}} \cdot \Delta \sigma=0
$$

The constitutive law, as in the examples discussed in Section 2 , is written in the form

$$
\begin{aligned}
& \nabla \\
& \sigma
\end{aligned}=\stackrel{L}{\sim}:
$$

where $D$ is the symmetric part of $\Gamma$ and $L$, is homogeneous of degree zero in $\mathrm{D}$. 
Employing (2.3) and (3.11) in conjunction with (3.13) and (3.14) yields

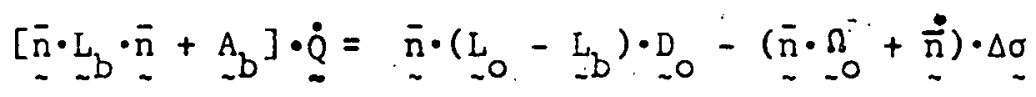

where

$$
A_{b}=\frac{l}{2}\left[-\bar{n}\left(\bar{n} \cdot \sigma_{b}\right)+\left(\bar{n} \cdot \sigma_{b} \cdot \bar{n}\right) I+\left(\tilde{n} \cdot \sigma_{b}\right) \dot{n}-\sigma_{b}\right]
$$

and $I$ is the unit tensor, $(I)_{i j}=\delta_{i j}$. The localization condition becomes

$$
\operatorname{det}\left\{\bar{n} \cdot L_{\sim} \cdot \underline{n}+\underset{\sim}{A}\right\}=0
$$

The alternative formulations (3.8) and (3.15) are fully equivalent and the choice between them is solely a matter of convenience (or taste). It does appear to us, however, that (3.8) is the more convenient formulation to employ when tracing a deformation history corresponding to a given initial imperfection whereas (3.15) is more suited to a bifurcation analysis. Specifically, if the material is initially homogeneous, the condition governing the onset of bifurcation into the localized band mode is that

$$
\left[\bar{n} \cdot L_{\sim} \cdot \bar{n}+\underset{\sim}{A_{0}}\right] \cdot \dot{\underline{Q}}=0
$$

have a nontrivial solution, where $\mathrm{A}_{0}$ is given by (3.16) with $\sigma_{b}$ replaced by $\sigma_{0}$, since prior to localization ()$_{b}=()_{0} \cdot$ This presumes that at localization the bifurcating field is associated with the same branch of the tensor of instantaneous moduli as the homogeneous field. 'For vertex-like yielding it seems possible that in some cases the bifurcating field could take advantage of the moduli associated with a different branch, as suggested by Rice [3].

We emphasize that neither the bifurcation analysis nor the analysis 
tracing the growth of an initial inhomogeneity require that the tensor of instantaneous moduli, $\underset{\sim}{K}$, be symmetric [3, 27]. However, if the moduli $\underset{\sim}{K}$ are symmetric (the symmetry of $L$ implying that of $K$ and vice versa only for an incompressible solid) a direct connection can be made between bifurcation into the band mode and loss of uniqueness in a specific boundary value problem, namely the problem of a solid subject to all.around displacement boundary conditions on its surface [26]. Presuming symmetry of the moduli and homogeneity of the deformation field, the band mode is the only bifurcation mode available under these restrictive boundary conditions and is often termed a "material" instability as opposed to "geometric" necking instabilities which these boundary conditions rule out.

Subject to the above conditions and, additionally, assuming incrementally linear material behavior (i.e., $\underset{\sim}{K}$ independent of $\dot{F}$ ), a linear stability analysis [3] shows that the onset of bifurcation into the band mode corresponds to the boundary between stability and instability for a displacement mode with a Fourier component having a polarization vector parallel to $\mathrm{n}$. Under less restrictive boundary conditions, "geometric"! instabilities (which may include arbitrarily short wavelength modes [23, 28]) invariably precede the band type localization considered here, but the material instability is still available, and sometimes, depending on specific circumstances, at overall deformations not greatly in excess of the geometric modes:

Although here a unified framework for analyzing localization instabilities in a wide class of materials has been presented, the actual onset of localization depends critically on the details of the constitutive description of the solid, as will be illustrated subsequently. 


\section{Results for Plastic Flow Localization}

The formalism for localization of the last section has been applied to the various constitutive models discussed in Section 2 . Consider first the classical Prandtl-Reuss model, eqs. (2.2 - 2.7). This emerges as a special case of a calculation by Rudnicri and Rice [2] and for localization the hardening rate must fall to the value

$$
h_{\text {crit }}=-2(1+v) G P_{I I}^{2}+O\left(\tau_{e}^{2} / G\right)
$$

where $P_{I I}$ is the internediate principal value of the plastic flow direction tensor $P$, namely

$$
P_{I I}=\sigma_{I I}^{\prime} / 2 \tau_{e}
$$

Since $\tau_{e}$ is typically a small fraction of $G$, the terms $O\left(\tau_{e}^{2} / G\right)$. are usually negligible and thus this nodel requires strain softening for localization in all deformation states except for plane strain $\left(P_{I I}=0\right)$, in which case $h_{\text {crit }} \approx 0$. The states most resistant to localization are those of axisymmetric extension or compression (or, equivalently, balanced biaxial tension) for which $P_{I I}= \pm 1 / 2 \sqrt{3}$ and

$$
\mathrm{h}_{\text {crit }} \approx-(1+v) \mathrm{G} / 6 \approx-0.2 \mathrm{G}
$$

The prediction of such strongly negative hardening rates for localization may be unrealistic for reasons that can be explored with the help of other constitutive models. Mevertheless, the results are suggestive of significant differences in ductility when plane strain and axisymmetric deformations are compared, e.g., Clausing [29]. In all cases the normal to the plane of localization is in the plane of the greatcst and least principal stresses, $\sigma_{I}$ and $\sigma_{\text {III }}$ respectively. For $\nu=0.3$ the angle $\theta$ between the normal 
and the $\sigma_{I I I}$ axis is $48.7^{\circ}, 45^{\circ}$, and $41.2^{\circ}$ for axisymmetric extension, plane strain (or pure shear), and axisymmetric compression respectively (Rudnicki and Rice [2]).

Now consider the effect of a yield vertex in the form modelled by eqs. (2.8 - 2.9). The calculation is rather complicated in general but simplifies when the material is taken to be elastically as well as plastically incompressible, $v=1 / 2$ : The result is most conveniently stated in terms of the moduli

$$
h^{\prime}=\frac{h}{1+h / G} \quad, \quad h_{1}^{\prime}=\frac{h_{1}}{1+h_{1} / G}
$$

which reduce to $h$ and $h_{1}$, respectively, for highily ductile materials where we expect $h \ll h_{1} \ll G$. In fact, $h^{\prime}$ is just the tangent modulus based on the total (rather than plastic) strain and, if the vertex modulus $h_{1}$ is identified as the plastic secant modulus, then $h_{1}^{\prime}$. is the secant modulus based on the total strain.

The critical conditions are established by Rice [3] for cases in which the vertex modulus is large enough to satisfy

$$
h_{1}^{\prime}>2\left(1-3 P_{I I}^{2}\right) h^{\prime} /\left(1-6 P_{I I}^{2}\right) \text {, }
$$

with $P_{I I}$ given by $(4.2)$. The inequality reads $h_{l}^{\prime}>2 h^{\prime}$ for plane strain states, and is satisfied for all deformation states if $h_{l}^{\prime}>3 h^{\prime}$. We expect the inequality to be satisfied for materials with light strain hardening. For example, if a pure power law stress-strain relation is satisfied, $\sigma_{e} \propto\left(\varepsilon_{e}\right)^{N}$, and if $h_{l}^{\prime}$ is interpreted as the secant modulus, then the inequality is satisfied for plane strain states whenever $N<1 / 2$ and for all states whenever if $<1 / 3$.

In all cases for which the inenuality $(4.4)$. is met $h_{\text {crit }}^{\prime}$ is the value 
of $h^{\prime}$ satisfying [3]

$$
\tau_{e}^{2}=12 P_{I I}^{2}\left(h_{l}^{\prime}-h^{\prime}\right)^{2}+4 h^{\prime}\left(h_{l}^{\prime}-h^{\prime}\right) \text {, }
$$

which includes a plane-strain resuit of Hill and Hutchinson [28] when we set $P_{I I}=0$. If we write $\because$ for the ratio, $h^{\prime} / h_{1}^{\prime}$ (noting that $M=N$ for pure power-law materials when the secant modulus definition is adopted for $h_{1}^{\prime}$ ), this can be rewritten as the critical condition

$$
h_{\text {crit }}^{\prime}=\frac{M \tau_{e}}{\sqrt{12 P_{I I}^{2}(1-M)^{2}+4 M(1-M)}},
$$

which reduces to

$$
\begin{gathered}
h_{\text {crit }}^{\prime}=\frac{\tau}{2} \sqrt{\frac{1}{1-i l}} \text { for plane strain } \\
h_{\text {crit }}^{\prime}=\tau_{e} \frac{M}{\sqrt{(1+3 M)(1-i)}} \text { for axisym. strain }
\end{gathered}
$$

We expect $y$ to have a range similar to that for the hardening exponent, say 0.05 to 0.5 , and thus we see from these expressions that localization occurs when $h_{c r i t}^{\prime}$ is some positive, but typically small, fraction of the equivalent shear stress $\tau_{e}$. Table 4.1 gives numerical values and we see that the critical hardening rate under plane strain conditions is always larger than for axi-symmetric conditions, indicating, the greater stability of the latter. For pure nower-law materials, $\sigma_{e} \sim \varepsilon_{c}^{N}$, and $h_{1}^{\prime}$ as the secant modulus (so $i 1=N$ ) the above results give the critical strains to localization (expressed in terms of both $\varepsilon_{e}$ and the greatest principal strain $\varepsilon_{I}$ ) of 


$$
\begin{aligned}
& \left(\varepsilon_{I}\right)_{\text {crit }}=\frac{\sqrt{3}}{2}\left(\varepsilon_{e}\right)_{\text {crit }}=\sqrt{N(1-H)} \text {, plane strain } \\
& \left(\varepsilon_{I}\right)_{\text {crit }}=\left(\varepsilon_{e}\right)_{\text {crit }}=\sqrt{(I+3 N)(1-N) / 3} \text {, axisym. strain. }
\end{aligned}
$$

These values are also shown in the table, as is the ductility ratio (based on strain to localization)

$$
\frac{\left(\varepsilon_{I}\right)_{\text {crit, pl. str. }}}{\left(\varepsilon_{I}\right)_{\text {crit, axisym. str. }}}=\sqrt{\frac{3 \mathrm{~N}}{1+3 \mathrm{~N}}}:
$$

The last exprassion is also plotted in Fig. 6 .

Clausing [29] reports a decreasing ductility ratio (based on fracture strains.) with increasing strength level for a series of seven structural steels; the ratio (based on $\varepsilon_{I}$ ) varies from 0.72 for a mild steel to 0.17 for a high strength ( $1770 \mathrm{MPa}$, or $248 \mathrm{ksi}$ ) steel. Clausing's data is summarized in Table 4.2. Indeed, comparing Tables 4.1 and 4.2, and assuming for the moment that the localization strain and fracture strain coincide, there are some important qualitative similarities. First, it is true as a general rule that the hardening exponent decreases with increasing strength level and thus the trend of the results in Table 4.1 and Fig. 6 seem consistent with Clausing's data. Also, it is the plane strain ductility, rather than the axisymmetric ductility, which is most affected by variations in the plastic flow properties. The comparison of this simple vertex model with Clausing's results is, in fact, encouraging enough that perhaps a great deal more effort should be directed to the further experimental and theoretical delineation of vertex effects and their role in localization.

In equating the strain to localization with that for fracture, we assume that conditions are such that a small amount of relative displacement 
For power-law hardening and $H=M$

\begin{tabular}{|c|c|c|c|c|c|}
\hline$M \equiv \frac{h^{\prime}}{h_{l}^{\prime}}$ & plane strain & $\begin{array}{l}/ \tau_{\mathrm{e}} \\
\text { axisym. str. }\end{array}$ & $\begin{array}{r}\left(\varepsilon_{I}\right) \\
\text { Dlane strain }\end{array}$ & axisym. str. & $\begin{array}{l}\text { ductility ratio } \\
\text { nlane/axisym. }\end{array}$ \\
\hline $\begin{array}{l}0.05 \\
0.1 \\
0.2 \\
0.3 \\
0.4\end{array}$ & $\begin{array}{l}0.115 \\
0.167 \\
0.250 \\
0.327 \\
0.408\end{array}$ & $\begin{array}{c}0.048 \\
0.092 \\
0.177 \\
0.260 \\
-\end{array}$ & $\begin{array}{l}0.218 \\
0.300 \\
0.400 \\
0.458 \\
0.490\end{array}$ & $\begin{array}{c}0.603 \\
0.624 \\
0.653 \\
0.666 \\
-\end{array}$ & $\begin{array}{c}0.361 \\
0.480 \\
0.612 \\
0.688 \\
-\end{array}$ \\
\hline
\end{tabular}

Table 4.1 Theoretical predictions of the critical hardening modulus ( $h_{\text {crit }}^{\prime}$ ) for localization as a function of ratio $M$ of tangent to vertex modulus. The critical strains for localization and the ductility ratio are calculated on the assumption that there is power-law hardening, $\sigma_{e} \sim\left(\varepsilon_{e}\right)^{N}$, and that the vertex modulus equals the secant modulus (i.e., for "deformation" theory) so that $\mathrm{M}=\mathrm{N}$.

$$
\left(\varepsilon_{I}\right) \text { Eract. }
$$

\begin{tabular}{|l|c|c|c|c|}
\multicolumn{1}{|c|}{ Material } & $\sigma_{\text {yield }}(\mathrm{MPa})$ & plane strain & axisym. str. & $\begin{array}{c}\text { ductility ratio } \\
\text { plane/axisym. }\end{array}$ \\
\hline $18 \mathrm{Ni}(250)$ & $1770(248 \mathrm{ksi})$ & 0.15 & 0.89 & 0.17 \\
$10 \mathrm{Ni}-\mathrm{Cr}-\mathrm{Mo}-\mathrm{Co}$ & 1300 & 0.36 & 1.16 & 0.31 \\
$18 \mathrm{Ni}(180)$ & 1270 & 0.42 & 1.00 & 0.42 \\
$\mathrm{HY}-130(\mathrm{~T})$ & 970 & 0.54 & 1.06 & 0.51 \\
$\mathrm{HY}-80$ & 610 & 0.86 & 1.22 & 0.70 \\
A302-B & $380 \quad:$ & 0.72 & 0.98 & 0.73 \\
$\mathrm{ABS}-\mathrm{C}$ & $280(39 \mathrm{ksi})$ & 0.75 & 1.04 & 0.72 \\
\hline
\end{tabular}

Table 4.2 Data on strain to fracture by Clausing [29], for seven structural steels arranged in order of decreasing strength level. 
over a concentrated band suffices to nucleate and grow an array of microcavities to coalescence. Whether or not this is so will be dependent on the material and, for a given material, may be strongly dependent on the prevailing stress state. For example, it seems possible that deformation could become inhomogeneous without fracture occurring in low triaxiality stress states, e.g., torsion, or for deformation under large hydrostatic pressure, even in materials for which fracture in plane strain tension is essentially coincident with localization.

Finally, we remark that all of Clausing's steels were materials of high ductility (at least in axisymmetric tension) and it is plausible that their stability against flow localization might be limited by some inherent feature of the piastic flow process, e.g., the vertex yield surface structure that arises from the crystalline basis for slip. In other alloys, or perhaps in the same alloys under more triaxially elevated stress states, it is possible that substantial ductility reductions could occur due to the nucleation and growth of microscopic cavities. It remains an open question in many systems as to whether ductile fracture arises from some instability of the plastic flow process, which then concentrates local strains and leads to cavity nucleation and growth to coalescence, or whether fracture is traceable instead to some instability brought about by the progressive micro-rupture process itself. To examine the matter further, we now consider the plastically dilatant material with pressure sensitive yielding, eq̧. $(2.10-2.11)$.

For this constitutive relation the critical hardening rate is given by Rudnicki and Rice [2] (we have done some algebraic rearrangement of their eq. (24) in light of their eq. (13)):

$$
\begin{aligned}
& v_{\text {arit }}=\left[\frac{1+y}{i(i-v)}(x-u)^{2}-\frac{1+v}{2}\left(1+\frac{a+u}{3}\right)^{2}\right] ; \\
& +\frac{(1+v)(\mu-u)}{12(1-v)} \sqrt{4-3 \Lambda^{2}}\left[\frac{1-2 \nu}{2} \Lambda+\frac{1}{2} \sqrt{4-3 \lambda^{2}}-\frac{1+\nu}{3}(\mu+\beta)+O\left(\tau^{2} / 3\right) .\right.
\end{aligned}
$$


Here $\Lambda$ (used in plase of the symbol $N$ of [2] to avoid confusion with the hardening exponent) is

$$
\Lambda=\sigma_{I I}^{\prime} / \tau_{e}
$$

and $\Lambda=-1 / \sqrt{3}$ for axisymmetric extension, 0 for a pure shear state of deviatoric stress, and $1 / \sqrt{3}$ for axisymmetric compression. The solution of (4.9) applies subject to certain restrictions on the size of $\mu$ and $B$ noted in [2]; when these are met the normal to the plane of localization lies in the I-III plane.

We observe first that when normality apolies (i.e., when $B=\mu$ ) $h_{\text {crit }}$ is non-positive for all stress states (neglecting the $O\left(\tau^{2} / G\right)$ terms); it vanishes when $\Lambda=-(\mu+\beta) / 3$. But when $\mu \neq \beta$, $h_{\text {crit }}$ can be positive for some deformation states.

An important special case is that of a plane strain state. From (2.11) for the plastic flow direction tensor?,

$$
P_{I I}=\sigma_{I I}^{\prime} / 2 \tau e+B / 3=\Lambda / 2+B / 3,
$$

so that $\Lambda=-2 B / 3$ for the case of plane strain. In this instance (4.9) reduces to

$$
\begin{aligned}
& h_{\text {crit }}=\frac{(1+\nu)^{2}}{18(1-v)}\left[(\mu-\beta)^{2} G\right. \\
& \left.\quad+(\mu-\beta) \sqrt{1-B^{2} / 3}\left(\frac{3}{1+v} \sqrt{1-\beta^{2} / 3}-\mu+\frac{3 v}{1+v} \beta\right) \tau_{e}\right]+O\left(\tau_{e}^{2} / G\right)
\end{aligned}
$$

This last expression may be applied to an aralysis of the strength differential effect as discussed in Section 2. Using (2.15) for $\mu$, assuming. $S D \ll 1$, and setting $B=0$, one finds

$$
h_{\text {crit }}=\frac{(1+v)^{2}}{24(1-v)}\left[(S D)^{2} G+\frac{\sqrt{3}}{1+v}(S D) \tau_{e}\right] \text {. }
$$


As an example, setting $S D=0.06, v=0.3$, using $6=32400: \mathrm{Pa}$ as appropriate to steel, and assuming that $\sigma_{e}\left(=\sqrt{3} \tau_{e}\right)=1400: 1 \mathrm{P}_{\mathrm{a}}$ $(\approx 200 \mathrm{ksi})$, one obtains

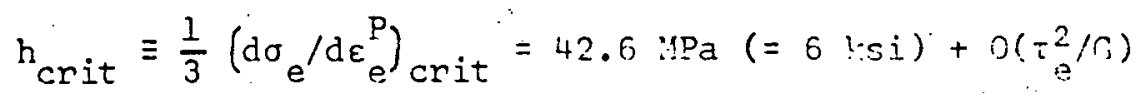

or, for comparison with entries in Table 4.1 hased on the vertex model, $h_{c r i t} / \tau_{e} \approx 0.05$. But for axisymmetric extension on compression values of $h$ crit computed from (4.9), for values of the parameters as above, $A=f e r$ little from that of eq. (4.i) for the classical Prandtl-Reuss model. It is not yet clear as to what the effects of small non-normality, as in the sn effect, and vertex yielding, would be if present concurrently.

The positive critical hardening rate that may reșult for states like plane strain, eq. (4.10), when $\mu \neq \beta$ seems to he typical of other cases with non-normality. Fon example, Asaro and Rice [30] discuss the localisation of plastic flow in ductile single crystals and observe that when there is nornomality, in the sense that stresses other than the scrmid resolved stress enter the criterion for continuing slip, localization can occus with a rositive slip system hardening rate. The implications of such Ldas son polycrystalis have not yet been fully explored.

As final examples, we consider the constitutive relation based on the Gurson model for porous solids, being cognizant of the cautionary romarks made regarding this model at the close of section 2. This relation has the same form as in $(2.10,11)$ and the localization condition is given as in (4.3), provided that the farameters $h, B$, and $\mu$ are defined as in eqs. (2.22 2.24). In these last equations it is to be recalled that $\bar{h}$ and $\bar{\sigma}$ are tha equivalent tensile rate of hardening and flow strenpth of the matrix naterial, 
$f$ is the void volume fraction, and $A$ and $B$ are parameters entering the expression (2.19) for the part of the increase of volume fraction $f$ arising from the nucleation of new voids. As remarked earlier, the macroscopic hardening rate $h$ can be negative and, when there is stress-dependent void nucleation $(B \neq 0), \mu$ can differ from $B$ so that deviations from normality occur.

We limit the analysis to plane strain conditions, so that $h_{\text {crit }}$ is given by (4.12). Further, in practical cases we expect $\beta$ and $\mu$ to be small compared to unity (exceptions may occur at high mean normal stress levels, e.g., in front of a sharp notch or crack) and aliso we assume that $f$ is small. In these cases we can simplify $(2.22-2.24)$, to read

$$
\begin{gathered}
h \approx \frac{1}{3} \bar{h}-\frac{1}{2} f c s \bar{\sigma}-\frac{1}{3} c \bar{\sigma} A \bar{h}, \\
\mu-\beta \approx \frac{1}{\sqrt{3}} c \bar{B} \bar{\sigma} .
\end{gathered}
$$

Further, we can write $\tau_{e}=\sigma_{e} / \sqrt{3} \approx \bar{\sigma} / \sqrt{3}$ in the same circumstances, and thus (4.12) becomes

$$
\frac{1}{3} \bar{h}-\frac{1}{2} f c s \bar{\sigma}-\frac{1}{3} c \bar{\sigma} A \bar{h}=\frac{1+v}{3 \sigma(1-v)}\left[\frac{1}{3}(c B \bar{\sigma})^{2} E+2 c B \bar{j}^{2}\right]
$$

at critical conditions, where $E$ is the elastic tensile modulus.

First we consider the case in which void nucleation is strain controlled,

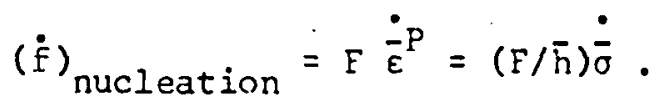

Evidently, $\Lambda=F / \bar{h}$ and $B=0$ for this case, and $F$ can be interpreted as the volume fracture of particles converted (effectively) to voids per unit of plastic strain. On the basis of Gurland's [18] data on spheroidized carbides in steel, Gurson $[12,17]$ suggests that $F \approx 0.3 \mathrm{~F}_{\mathrm{up}}$, where $f_{\text {up }}$ is the volume fraction of unbroken carbide particles. In terms of $F$, the critical condition becomes 


$$
(\bar{h} / \bar{\sigma})_{\mathrm{crit}} \approx \frac{3}{2} \mathrm{f}(\cosh \Sigma)(\sinh \Sigma)+F(\cosh \Sigma)
$$

(recall that $\Sigma$ denotes $\sigma_{k k} / 2 \bar{\sigma}$ ). According to this model, and in view of the preceding remarks concerning $F$, the critical $\vec{h} / \bar{\sigma}$ value is predicted to be of the order of the void volume fraction $f$ or of the order of the volume fraction of uncracked particles, at least for states of only modest stress triaxiality.

In the case of stress dependent nucleation we may write

$$
(\dot{\mathrm{f}})_{\text {nucleation }}=\mathrm{K}\left(\dot{\bar{\sigma}}+\dot{\sigma}_{k \mathrm{k}} / 3\right) / \bar{\sigma}
$$

so that $K$ is the volume fracture of particles converted to voids per unit fractional increase in stress. Thus $A=B=K / \bar{\sigma}$ and the critical condition is

$$
\begin{aligned}
& (\bar{h} / \bar{\sigma})_{\text {crit }}=\frac{1}{1-K(\cosh \Sigma)}\left\{\frac{3}{2} f(\cosh \Sigma)(\sinh \Sigma)\right. \\
& \vdots \\
& \vdots \\
& \left.\quad+\frac{1+v}{6(1-v)} K(\cosh \Sigma)\left[1+\frac{E K(\cosh \Sigma)}{6 \bar{\sigma}}\right]\right\}
\end{aligned}
$$

We note that in cases for which void nucleation takes place over a comparatively narrow range of stress, $k$ can be several times the volume fraction of void nucleating particles and then the above expression can predict that rather large values of $\bar{h} / \bar{\sigma}$ are required for stability against localization. For example, choosing $\Sigma=\sqrt{3} / 2$ as appropriate to the plane strain tension test (without necking effects) and $v=0.3$,

$$
(\bar{h} / \bar{\sigma})_{\text {crit }}=\frac{1}{1-1.40 \mathrm{~K}}\left\{2.06 \mathrm{f}+0.43 \mathrm{~K}+0.10 \mathrm{~K}^{2} \mathrm{E} / \bar{\sigma}\right\}
$$

and when we consider the current void volume fraction $f$ to be negligible compared to $K$ and set $F / \bar{\sigma}=300 \cdot(\mathrm{e} . \mathrm{g} \cdot, \bar{\sigma}=100 \mathrm{ksi} \approx 700 \mathrm{MPa}$ in steel $)$, 
we get

$$
(\bar{h} / \bar{\sigma})_{\text {crit }}=0.007,0.10 \text {, and } 0.40
$$

for

$$
K=0.01,0.05 \text {, and } 0.10
$$

respectively.

All the results cited thus far pertain to the onset of bifurcation. However, there may be significant sensitivity to small initial non-uniformities of material properties and this aspect of the problem is not yet well explored in general. However, Yamamoto has adopted an approach similar to that outlined in Section 3 for a material described by the Gurson constitutive model (2.10-11 and $2.22-24)$ but in which the initial void volume fraction, $f_{0}$, is slightly larger in a planar slice of material than elsewhere. Yamamoto neglects the nucleation terms $(A=B=0)$ so that instability results only from the porosity increase due to the progressive growth of existing voids.

Some of Yamamoto's results are shown in Fig. 7, for which an initial porósity of $f_{0}=0.01$ is assumed outside the imperfect zone, and the strain $\varepsilon_{I}$ to failure is shown as a function of the initial porisity, $f_{\text {imp.,o }}$, inside the imperfection. The calculations were done for axisymmetric and plane strain tension cases with. $\sigma_{\text {III }}=0$ in all cases. The latter means that the induction of triaxial tension stresses, which occurs as part of the necking process in experiments, was neglected in the calculation. This is a serious defect since, as has been seen, the Gurson dilational plasticity equations are strongly sensitive to the mean normal stress.

Yamamoto's calculations employed the power hardening relation

$$
\frac{\bar{\sigma}_{j}}{\sigma_{y}}=\left[\frac{\bar{\sigma}}{\sigma_{y}}+\frac{3 E \bar{\varepsilon}^{-P}}{2(1+v) \sigma_{y}}\right]^{N}
$$


for the matrix material, with $v=0.3$ and $\sigma_{\mathrm{y}} / E=0.003$. Results are șhown in Fig. 7 for two hardening exponents, $i=0.1$ and 0.2 , and in each case these correspond to a numerical search over orientation angles of the slice to give the least ductility. Although not shown on the figure, Yamamoto also calculates the results for $f_{0}=0$ outside the imperfection and we find that these, when plotted against $f_{\text {imp,o }}$, give results which are very similar to those shown. The results indicate that the localization condition for a void-containing material is strongly sensitive to nonuniformities in the porosity distribution. We will see similar effects for sheet metals in the following section. Yamamoto also observes that for a given size imperfection the ductility ratio (plane strain to axisymmetric) increases with decreasing hardening exponent. For example, in calculations for which the size of $f_{i m p, 0}-f_{0}$ varies from 0.01 to 0.05 , and for $f^{0}=0$ to 0.01 , Yamamoto finds a ductility ratio of from 0.13 to 0.20 for $N_{r}=0.1$ and from 0.26 to 0.30 for $N=0.2$. These seem somewhat low compared to experimental results, but the inclusion of necking effects in the analysis would, presumably, lower the $\left(\varepsilon_{I}\right)_{\text {crit }}$ for axisymmetric extension: more than for plane strain (since far. more of the strain to failure shown in Fig. 7 occurs after necking for axisymmetric conditions), and this could have the effect of raising the predicted ductility ratios somewhat. This remains a topic in need of further examination. 


\section{Localized Neckinĝ in Biaxially Stretched Sheets}

The mathematical theory of localized necking in thin sheets is a precise two dimensional analog of the three dimensional theory of flow localization outlined in Section 3. In that development one need only replace $\checkmark$ and $\sigma$ by the nominal and true membrane forces, respectively. Indeed, many of the basic principles of the theory were first elucidated within this two dimensional plane stress context $[24,32]$.

Hill [32] analyzed bifurcations corresponding to localized necking using classical (smooth yield surface, normality) rigid plastic theory. As is well known, this analysis predicts that local necking will not occur in a uniform sheet subject to positive biaxial stretching. Since both practical experience [33-35] and experimental tests [36-39] demonstrate that thin sheets subject to positive biaxial tension can fail by a process of localized necking, there has been considerable interest in attempting to resolve this "paradox". One approach, initiated by Marciniak and Kuczynski [24] (hereafter referred to as $M-K$ ), postulates an initial inhomogeneity in the sheet in the form of a localized thickness reduction. This instigates necking by precipitating a drift of the strain state in the neck toward plane strain, while the remainder of the sheet undergoes proportional loading.

Azrin and Backofen [36] carried out experiments aimed at testing the M-K model of localized necking and found that the magnitude of the assumed thickness reductions required to give theoretical predictions of the limit strain, that is the imposed strain at the onset of localized necking, in line with those observed experimentally were much larger than actually measured in the test specimens. Furthermore, although the experiments did 
show a drift of the strain state in the neck toward plane strain, the . dependence of the limit strain on the imposed strain ratio was qualitatively different from that predicted by the $M-K$ model for most of the materials tested. Specifically, for an isotropic material (and most of the materials tested in [36] appear not to have been significantly anisotropic), the $M-K$ analysis predicts that the limit strain should increase with increasing biaxiality, whereas experimentally, for a number of materials, the limit strain was found to be nearly independent of the imposed strain ratio or actually to decrease somewhat with increasing biaxiality.

A different line of attack was initiated by Storen and Rice [7] who showed that a simple model of a material with a vertex on its yield surface, namely a finite strain version of the simplest deformation theory of plasticity, does predict a bifurcation corresponding to localized necking, in biaxial tension. The limit strains obtained by means of this analysis are qualitatively more in accord with experiment than those given by the $M-K$ analysis. In particular, for a material exhibiting a pure power law uniaxial hardening curve of the form

$$
\sigma=K \varepsilon^{N}
$$

where $\sigma$ is the true stress and $\varepsilon$ the logarithmic strain in uniaxial tension, Stören and Rice's [7] analysis gives limit strains that monotonically decrease with increasing biaxiality for high hardening materials, $N>\frac{1}{3}$.

Although, in [24] as well as in a number of subsequent investigations, an initial thickness inhomogeneity was employed, this imperfection was 
assumed equivalent to a local variation in material properties and was not necessarily intended to be regarded as a literal thickness reduction. In some recent work $[40,41]$ an actual difference in material properties in the incipient neck has been accounted for within the $M-K$ framework. In particular, in [40] Needleman and Triantafyllidis have employed the $M-K$ model in conjunction with Gurson's [12, 13] constitutive relation for porous plastic materials. In [40], as in [31], void nucleation is neglected $(A=B=0$ in $(2.19))$.

Fig. 8 displays limit strains as a function of imposed strain|ratio, $\rho$, where

$$
\rho=\varepsilon_{2} / \varepsilon_{1}
$$

and $\varepsilon_{1}$ and $\varepsilon_{2}$ are, respectively, the major and minor principal in-plane logarithmic strains. The solid curves are the deformation theory results of [7], while the dashed curves are for the void growth model of [40], in which an increased initial void concentration, $\Delta f_{0}$, plays the role of the initial imperfection. Curves are displayed for various values of the strain hardening exponent in (5.1). It is important to note that in the porous plastic material model this strain hardening exponent pertains to the matrix material and not to the void matrix aggregate.

Both theories agree in predicting that for high hardening materials the limit strain will decrease or remain nearly constant with increasing biaxiality whereas for lightly hardening materials the limit strain will increase with increasing biaxiality. By suitably adjusting parameter values, the difference between the predictions of the two theories can be made negligible. This is, of course, a somewhat arbitrary exercise. The significant point is that the analyses in [7] and [40] suggest 
that both vertex effects and the weakening induced by incipient ductile. rupture provide plausible mechanisms to account for the observed shapes of forming limit diagrams. In essence, the deformation theory model attributes the onset of localized necking to the discrete crystallographic nature of slip whereas the void growth model ties localized necking to the (not well understood) factors responsible for the initiation of ductile rupture.

of course, vertex effects and incipient ductile fracture are not the only possible physical mechanisms that could be responsible for triggering localized necking. In Fig. 9 (adopted from [40]) the Forming limit curves resulting from various types of inhomogeneities are compared. The curves marked $\left(\sigma_{y}^{B} / \sigma_{y}^{A}\right)=0.99$, correspond to a one percent yield stress reduction in the neck with all other initial values being identical inside (region $B$ in Fig. 9) and outside (region A in Fig. 9) the neck. Similarly, the curves marked $\left(t^{B} / t^{A}\right)_{0}=0.99$, correspond to a one percent initial thickness reduction in the neck with all other initial values being identical inside and outside, and so on. With the exception of the curve marked $\Delta f_{0}=0.01$, the initial void concentration both inside and outside the neck was taken to be identically zero. With an initial void concentration of zero the Gurson constitutive equation reduces to the Prandtl-Reuss equations and, therefore, the void concentration remains zero. The inhomogeneity corresponding to $\left(\mathbb{N}^{B} / N^{A}\right)=0.99$, gives qualitatively the same behavior as the model incorporating void growth. This is not entirely unexpected since the principle effect of void growth is to increasingly decrease the stiffness of the void inatrix aggregate. In contrast, the forming limit curves 
for $\left(\sigma_{y}^{B} / \sigma_{y}^{A}\right)=0.99$ and $\left(t^{B} / t^{A}\right)_{0}=0.99$ monotonically increase with 0 . Un the other hand with $N^{\Lambda}=0.25$, all the forming limit curves have the same general shape [40].

These results have some bearing on the idea of an "equivalent thickness imperfection." In previous calculations of forming limit diagrams a thickness imperfection was employed as the inhomogeneity and it was hypothesized that this was representative of microstructural inhomogeneities. The results in [40] show that this hypothesis is not necessarily appropriate for high hardening materials. These results also indicate that any microstructural inhomogeneity that has the effect of continually decreasing the effective strain hardening exponent would be expected to give qualitatively similar forming limit diagrams to the void growth model. For example, in precipatation hardened solids there could be some local region of the sheet which is hardened less than the surrounding material.

This (somewhat obliquely) introduces the important question of the length scale over which it is reasonable to assume inhomogeneities act. of course, the deformation theory results of [7], being based on a bifurcation analysis do not require the assumption of an initial inhomogeneity. However, for an M-K type model, this question is a crucial one. Recent work [42] has indicated that the assumption of plane stress is an appropriate one for deformation wavelengths long with respect to the sheet thickness. Thus in the $M-K$ model, the inhomogeneity must be assumed to be one acting over a length scale of at least several sheet thicknesses. If the inhomogeneity is truly local, of the order of the sheet thickness or less, then a plane stress analysis, such as that in [40], may significantly overestimate the rate of growth of the inhomogeneity [42, 43]: 
Experimental studies directed toward characterizing the type, magnitude and spatial distribution of the inhomogeneities to be expected in sheet materials would be most valuable. As illustrated in Fig. 8, by arbitrarily choosing values of the characterizing parameters for sheet materials, theories based on very different physical mechanisms can yield virtually identical forming limit diagrams. Experimental guidance is required to determine what reasonable values of parameters are. Hopefully, coupled with theoretical analyses of relevant constitutive models, such studies would enable the dominant physical mechanisms responsible for triggering localized necking (which may very well be material dependent) to be identified.

Additionally, direct examination of the material in the neck, at various stages of necking, for evidence of incipient ductile rupture would indicate whether or not this mechanism plays a significant role. Here, it would be particularly important to include specimens with well developed necks, since in [40] it was found that most void growth takes place in the latter stages of necking.

The discussion so far has presumed that the plane stress local necking mode is the one that limits ductility. Although consideration here is focussed on thin sheets, it is by no means certain that a three dimensional band mode, of the type discussed in the previous section, is ruled out." Somewhat curiously, within the context of the three dimensional theory, the localized necking mode corresponds to a "geometric" instability and not a "material" instability. Whatever the underlying physical mechanism, there is the possibility of the deformation in the necked down region becoming significantly large to precipitate three dimensional flow localization 
(or to activate a "geometric" instability mode not accounted for in the plane stress theory $[23,28])$. The three dimensional localized necking mode, representing an actual material instability, provides an inherent limit, regardless of sheet thickness or other geometric effects, to ductility. Indeed, in the plane strain tension test, e.g. [29], ductility is often found to be limited by highly intense band type localizations, sometimes preceeded by only a small amount of growth of the diffuse "geometric" necking mode. 


\section{Concluding Remarks}

The description of localization as a material instability appears to provide a useful framework for analyzing both three dimensional "shear band" localizations and localized necking in ductile metal sheets (when these can be appropriately treated as two dimensional continua). In both contexts, the predicted onset of localization depends critically on the assumed constitutive description. The effects on localization of several deviations from the classical Prandtl-Reuss model have been illustrated. Significant constitutive features not explicitly dealt with here that can be accommodated within the theory include anisotropy, which is particularly important in the sheet metal context, and material rate dependence (which necessitates an analysis with an initial imperfection).

Encouraging results have been obtained from a simple model of a solid with a vertex on its yield surface, both regarding the ratio of plane strain and axisymmetric ductilities and forming limit diagrams. Hore or less similar results can also be obtained from a simple model of a solid weakened by micro-cavities. Both these constitutive features merit improved modelling in relation to the detailed mechanisms of deformation. A more thorough theoretical and experimental illumination of these aspects of material behavior could shed considerable light on the circumstances under which an inherent instability of the plastic flow process or progressive micro-rupturing is responsible for limiting ductility. 


\section{Acknowledgement *}

Support from the Energy Research and Development Administration through contract E(11-1)3084 and the National Science Foundation through grant EivG76-16421. is gratefully acknowledged. 


\section{References}

1. R. Hill, The liathematical Theory of Plasticity, Oxford (1950).

2. J.H. Rudnicki and J.R. Rice, "Conditions for the localization of deformation in pressure-sensitive dilatant materials", J. Mech. Phys. Solids, 23, 371-394 (1975).

3. J.R. Rice, "The localization of plastic deformation", in Theoretical and Applied Iechanics (Proc. 14th IUTAll Congress), ed. W.T. Koiter, Vol. I, North-Holland, 207-220 (1976).

4. R. Hill, "The essential structure of constitutive laws for metal composites and polycrystals", J. Mech. Phys. Solids, 15, 79 (1367).

5. T.H. Lin, "Physical theory of plasticity", Advan. Appl. Mech., 11, 255311 (1971).

6. J.W. Hutchinson, "Elastic-plastic behaviour of polycrystalline metals and composite", Proc. Roy. Soc. Lond., A139, 247-272 (1970).

7. S. Stören and J.R. Rice, "Localized necking in thin sheets", J. Mech. Phys. Solids, 23, 421-441 (1975).

8. J.L. Sanders, "Plastic stress-strain relations based on linear loading functions," Proc. 2nd U.S. Nat. Congr. Appl. Viech, 455-460. (1954).

9. J.W. Hutchinson and K.W. Neale, "Sheet necking - II. Time-independent behavior", this volume.

10. H.A. Spitzig, R.J. Sober and O. Richmond, "Pressure dependence of yielding and associated volume expansion in tempered martensite", Acta Net., 23, 885 (1975).

11. W.A. Spitzig, R.J. Sober and 0. Richmond, "The effect of hydrostatic pressure on the deformation behavior of maraging and $I Y-80$ steels and its implication for plasticity theory", let. Trans. 7A, 1703-1710 (1976).

12. A.L. Gurson, "Plastic flow and fracture behavior of ductile materials incorporating void nucleation, growth, and interaction", Ph.D. Thesis, Brown Univ., (available from Univ. Microfilms, Ann Arbor, Mich.), (19775).

13. A.L. Gurson, "Continuum theory of ductile rupture by void nucleation and growth: Part I - yield criteria and flow rules for porous ductile materials", Trans. ASilE, J. Engr. Mat'1. Tech., 99, 2-15 (1977).

14. F.A. McClintock, "A criterion for ductile fracture by the growth of holes", Trans. ASME, J. Anol. Mech., 35. 363-371 (1968).

15. J.F.W. Bishop and R. Hill, "A theory of the plastic distortion of a polycrystalline aggregate under combined stress", Phil. Mag., 42, 414-427 (1951). 
16. C.A. Berg, "Plastic dilation and void interaction", in Inelastic Behavior of Solids, eds., M.F. Kanninen et al., McGraw-Hill, 171-209 (1970).

17. A.L. Gurson, "Porous rigid-plastic materials containing rigid inclusions yield function, plastic potential, and void nucleation", in Fracture 1977, ed. D.M.R. Taplin, Vol. 2, 357-364 (1977).

18. J. Gurland, "Observations on the fracture of cementite particles in a spheroidized 1.05\% C steel deformed at room temperature", Acta. Met., 20,735-741 (1972).

19. A.S. Argon, J. Im and A. Needleman, "Distribution of plastic strain and negative pressure in necked steel and copper bars", Met. Trans. 6A, 815-824 (1975).

20. A.S. Argon and J. Im, "Separation of second phase particles in spheroidized 1045 steel, Cu-0.6 pet $\mathrm{Cr}$ alloy, and maraging steel in plastic straining", !et. Trans. 6A, 839-851 (1975).

21. A.S. Argon, J. Im and R. Safoglu, "Cavity formation from inclusions in ductile fracture", Met. Trans., 6A, 825-837 (1975).

22. J.R. Rice and M.A. Johnson, "The role of large crack tip geometry changes in plane strain fracture, in Inelastic Behavior of Solids, eds., M.F. Kanninen et al., McGraw-Hill, 64l-668 (1975).

23. A. Needieman and V. Tvergaard, "Necking of biaxially stretched elasticplastic circular plates", J. Mech. Phys. Solids, 25, 159-183 (1977).

24. Z. Marciniak and K. Kuczynski, "Limit strains in the process of stretch forming sheet metal", Int. J. Hech. Sci., 9, 609-620 (1967).

25. J. Hadamard, Lecons sur la Propagation des Ondes et les Equations de L'Hydrodynamique, Paris, Chp. 6 (1903).

26. ‥ Hill, "Acceleration waves in solids", J. Mech. Phys. Solids, 10, $1-16$ (1962).

27. J. Mandel, "Conditions de stabilite et postulat de Drucker", in Rheology and Soil Mechanics, eds.., J. Kravtchenko and P.M. Sirieys, SpringerVerlag, 58-68 (1966).

28. R. Hill and J.W. Hutchinson, "Bifurcation phenomena in the plane tensile test", J. Mech. Phys. Solids, 23, 239-264 (1975).

29. D.P. Clausing, "Effect of plastic strain state on ductility and toughness", Int. J. Fract. Mech., $\underline{6}, 71-85$ (1970).

30. R.J. Asaro and J.R. Rice, "Strain localization in ductile single crystals", J. liech. Phys. Solids, (in press).

31. H. Yamamoto, "Conditions for shear localization in the ductile fracture of void-containing materials", Brown University Report E(II-1)3084/50, April (1977), submitted for publication. 
32. R. Hill, "On discontinuous plastic states, with special reference to localized necking in thin sheets", J. Mech. Phys. Solids, 1, 19-30 (1952).

33. S.P. Keeler, "Understanding sheet metal formability"; Machinery, 74, lios. 6-11, February-July (1968).

34. S.S. Hecker, "Simple technique for determining forming limit curves", Sheet Metal Industries, 671-675 (1975).

3:5. S.S. Hecker, "Formability of aluminum alloy sheets", Trans. ASME.

J. Engr. Mat. Tech., 97, 66-73 (1975).

36. M. Arzin and W.A. Backofen, "The deformation and failure of a biaxially stretched sheet", Met. Trans., I, 2857-2865 (1970).

37. A.K. Ghosh and S.S. Hecker, "Stretching limits in sheet metals; in-plane versus out-of-plane deformation", Het. Trans., 5, 2161-2164 (1974).

38. A.K. Ghosh and S.S. Hecker, "Failure in thin sheets stretched over rigid punches", Metall. Trans., 6A, 1065-1074 (1975).

39. M.J. Painter and R. Pearce, "Instability and fracture in sheet metal", J. Phys. D: Appl. Phys., 7, 392-1002 (1974).

40. A. Heedleman and $N$. Triantafyllidis, "Void growth and local necking in biaxially stretched sheets", Trans. ASME, J. Engr. Mat. Tech., (in press).

41. A. Needleman, "Necking of pressurized spherical membranes", J. Hech. Phys. Solids, 24, 339-353 (1976).

42. J.H. Hutchinson, K.H. Neale and $\Lambda$. Needleman, "Sheet necking - I. Validity of plane stress assumptions of the long-wavelength approximation", this volume.

43. E.J. Appleby and O. Richmond, "Stretchability of wavy sheet", to be published.

44. T.Y. Thomas, "Plastic flow and fracture in solids", Academic Press (1961).

45. S.B. Batdorf, "Theories of plastic buckling", J. Aeronaut. Sci., 16, 405-408 (1949).

46. J.W. Hutchinson, "Plastic buckling", Advan. Appl. Mech., 14, 67-144 (1974).

47. S.B. Batdorf and B. Budiansky, "A mathematical theory of plasticity based on the concept of slip", NACA TN No. 1871 (1949). 
Figure Captions

1. Stress-strain curve showing the tangent modulus $h$ and the secant modulus $h_{i}$.

2. Schematic representations of yield surfaces in deviatoric stress space, showing the directions of the deviatoric stress rate $\stackrel{7}{\sigma}$, and the corresponding plastic strain rate for (a) Flow theory with a smooth yield surface and (b) Flow theory with a vertex at the current state; a hypothetical smooth yield surface passing through the current state is also shown.

3. Sketch of a yield surface illustrating the geometric interpretations of the pressure sensitivity factor $\mu$ and the dilatancy factor $\beta$.

4. Sketch illustrating the dependence of the yield function (2.16) on the mean normal stress for increasing volume fractions of voids, $f$.

5. Illustration of an element of a band in (a) The reference configuration where the area is $A$, and the normal is $n$ and (b). The current configuration where the area is $\bar{A}$ and the normal is $\bar{n}$.

6. Ratio of plane strain ductility to axisymmetric ductility as a function of the strain hardening exponent $N$, as predicted by the vertex model with the assumption that the vertex modulus equals the secant modulus.

7. Curves of the critical strain for localization as a function of the initial void concentration in the band for plane strain tension and axisymmetric tension, from Yamamoto [31]. 
$-45-$

8. A comparison of the forming limit curves predicted by the vertex model (solid curves) and the void growth model (dashed curves). Here, $\Delta f_{0}$ is the increased initial porosity in the neck. Based on [7] and [40].

9. A comparison of the predicted forming limit curves resulting from various types of initial inhomogeneities with strain hardening. exponent $N^{A}=0.67$. Here, ()$^{A}$ refers to quantities outside the neck and ()$^{B}$ refers to quantities inside the neck. Adopted from $[40]$. 


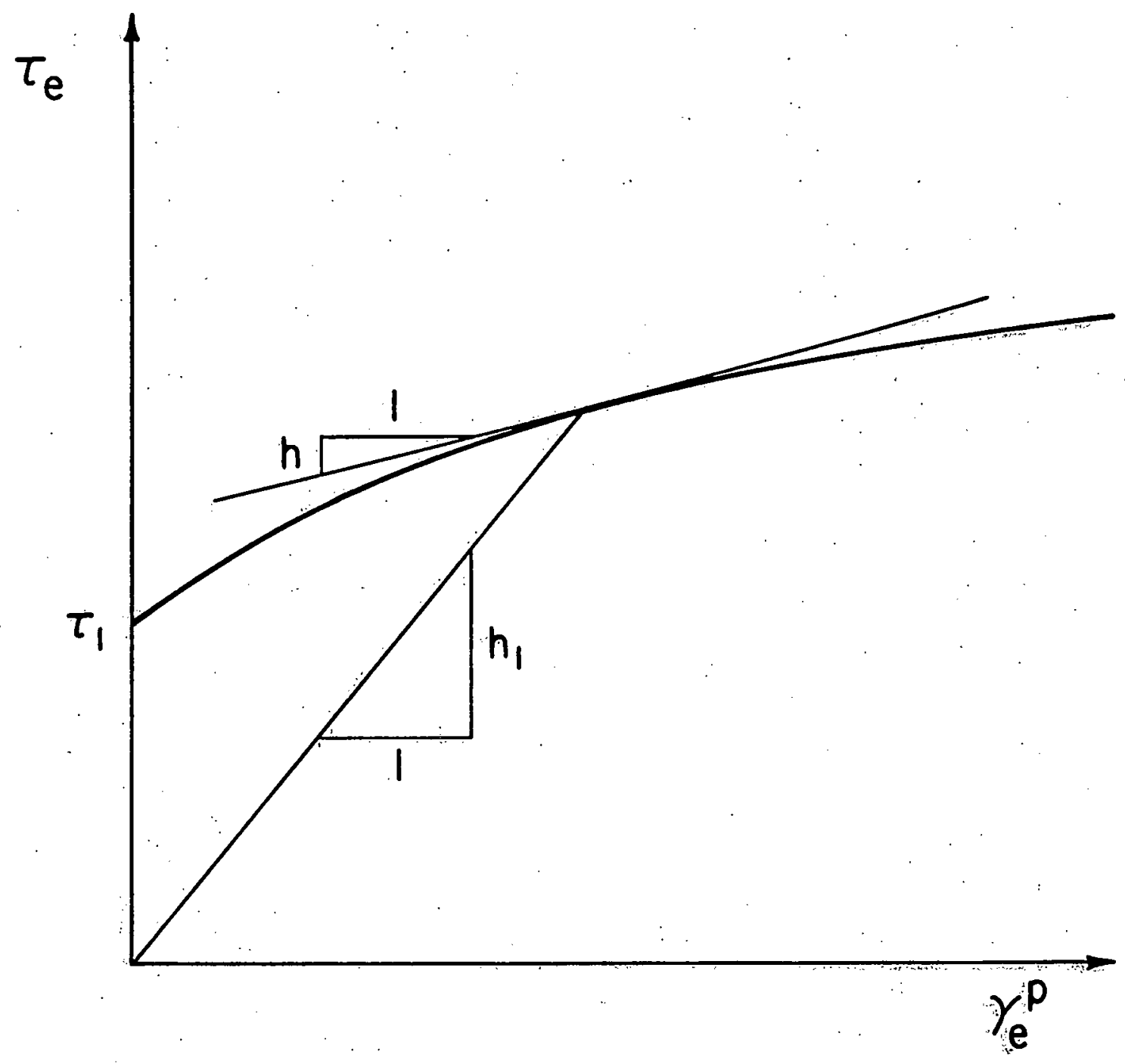

FIGURE I 

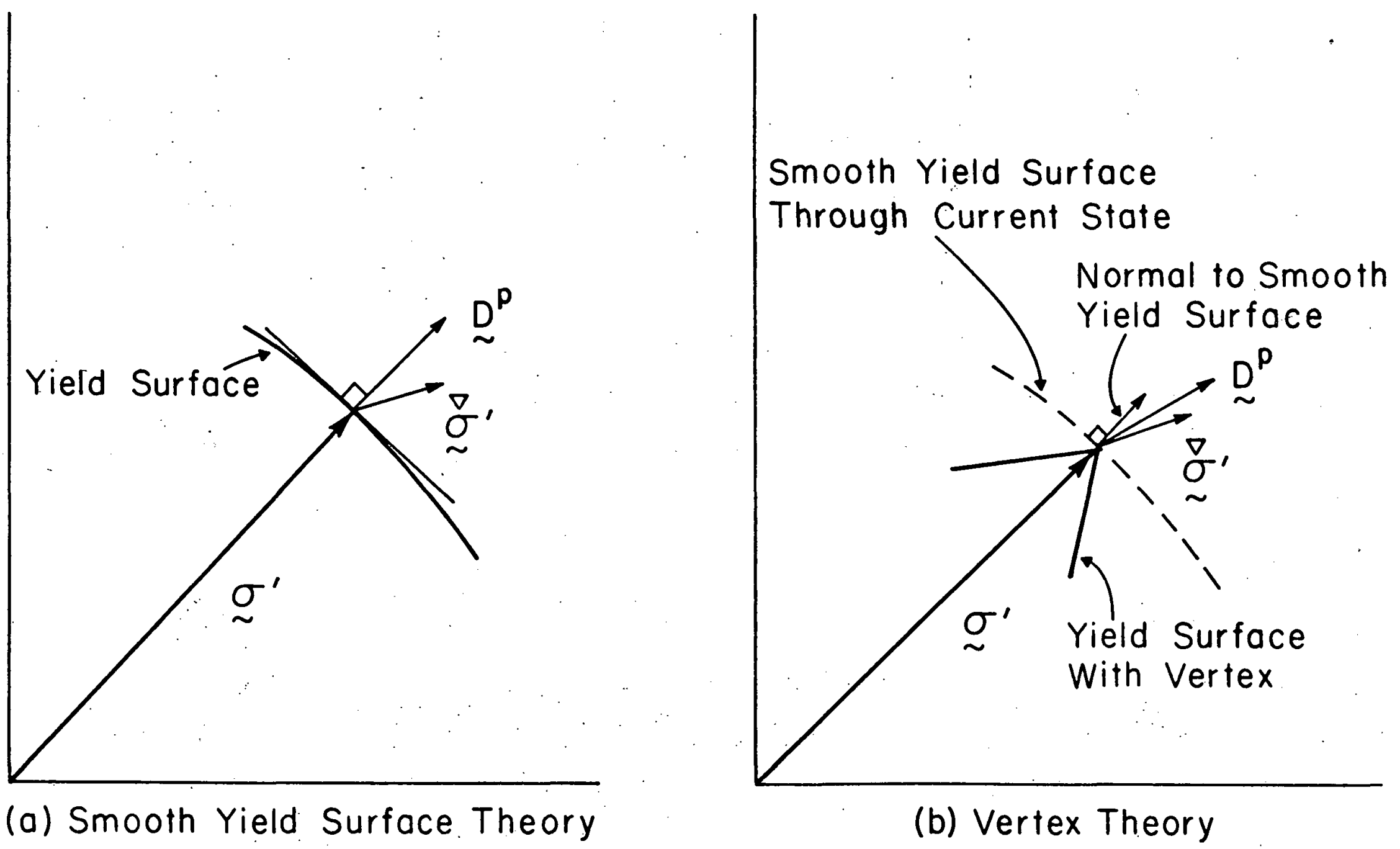

(b) Vertex Theory

FIGURE 2 


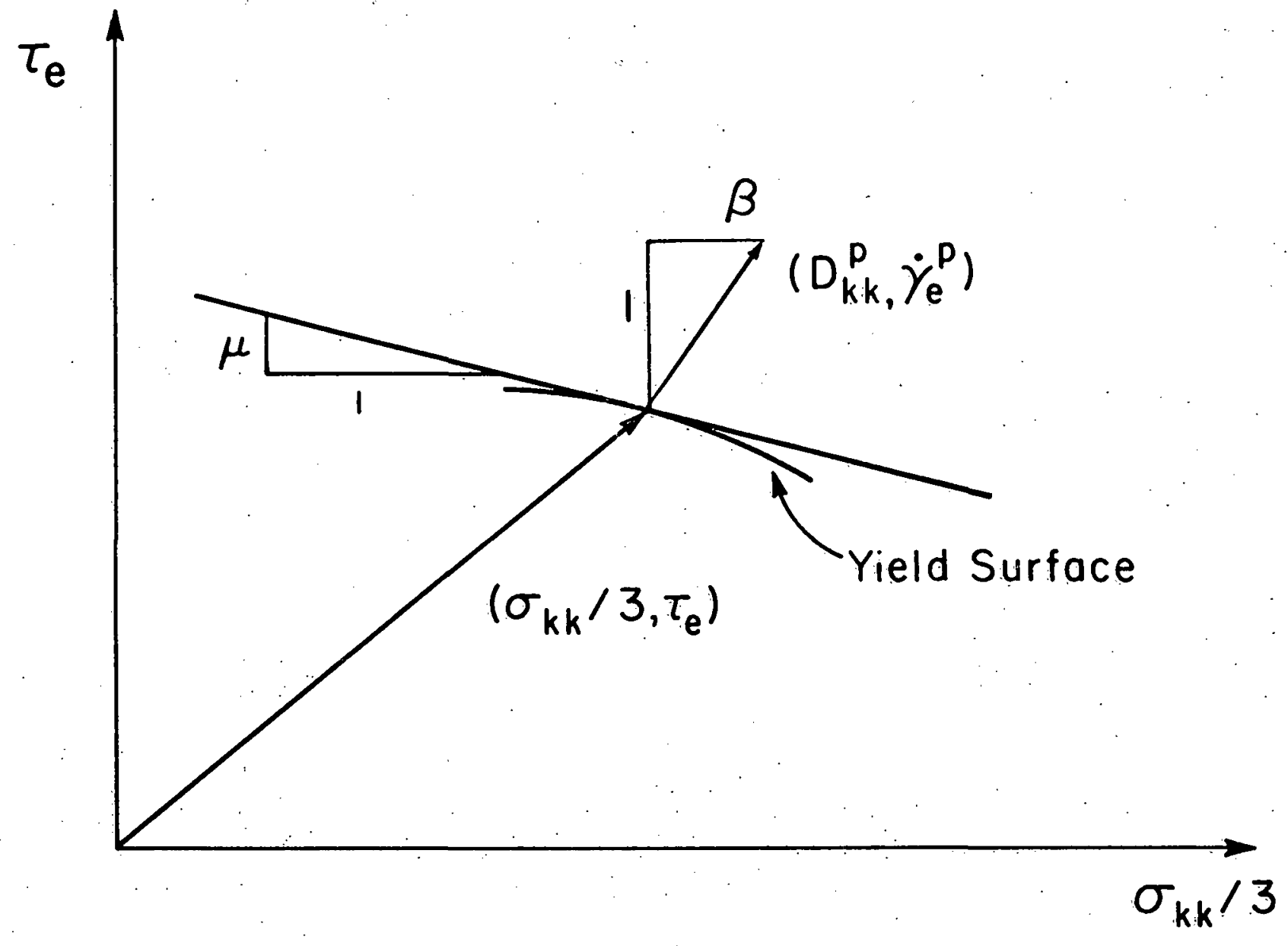

FIGURE 3 


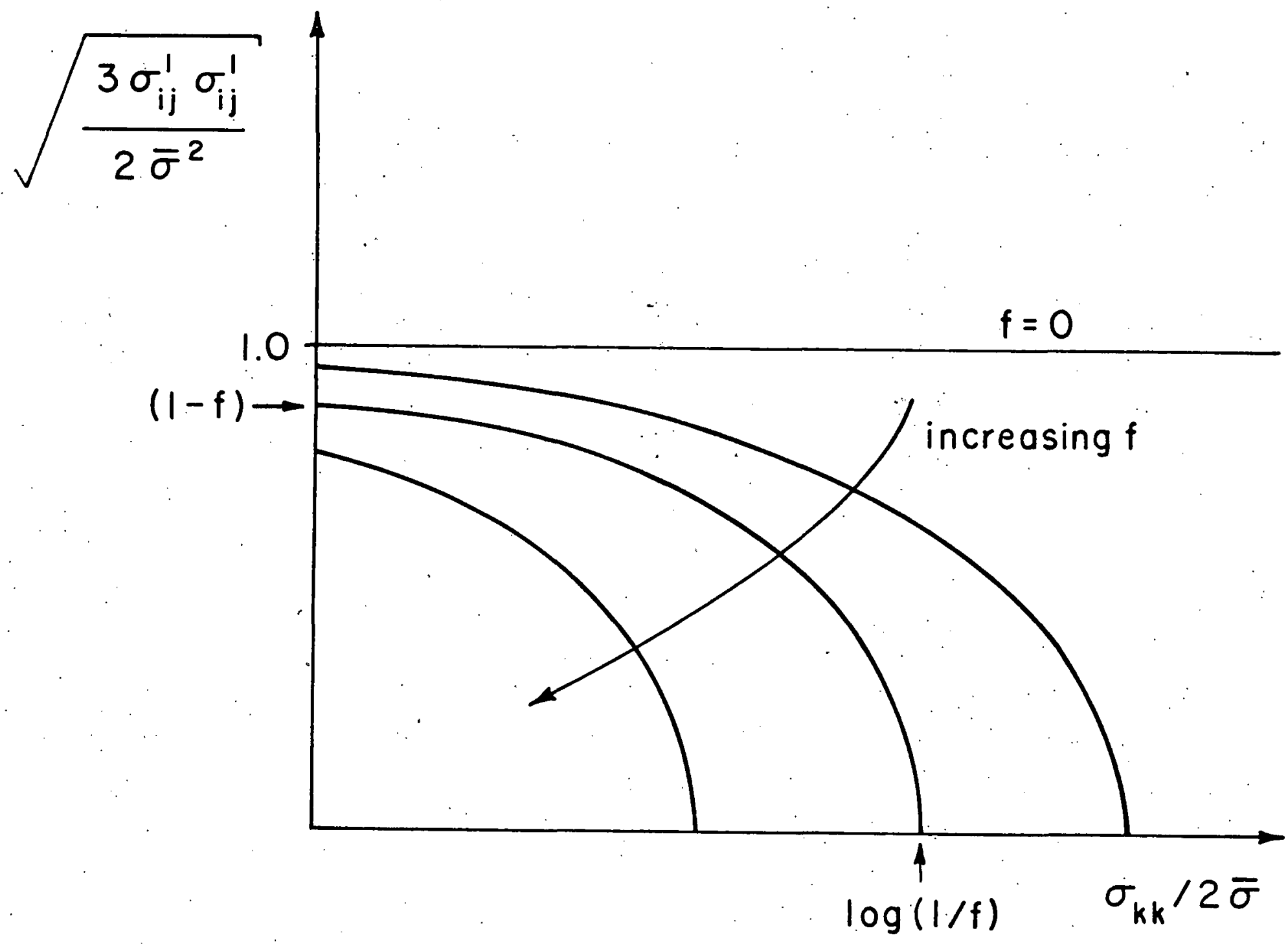

FIGURE 4 

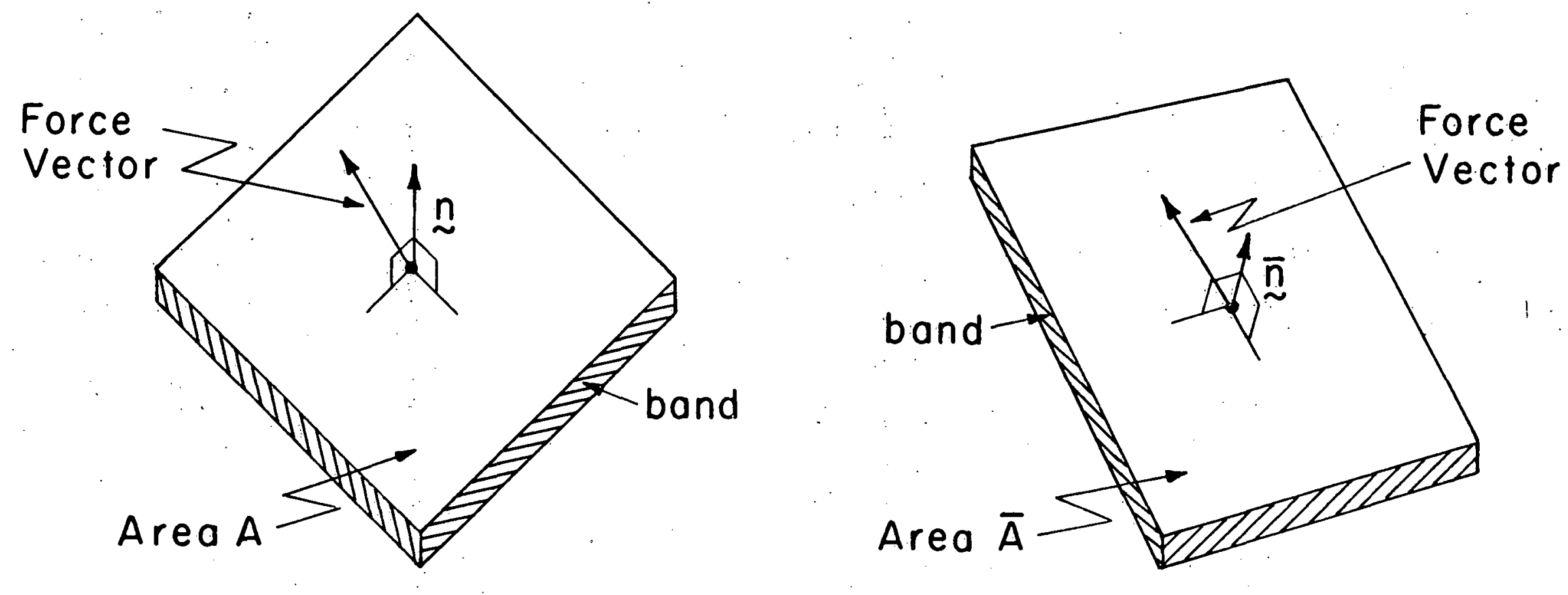

$$
\text { Force Vector }=(\underset{\sim}{n} \cdot \underset{\sim}{s}) A=(\underset{\sim}{\bar{n}} \cdot \sigma) \bar{A}
$$

a) Reference Configuration

b) Current Configuration

FIGURE 5 
PLANE STRAIN DUCTILITY/AXISYMETRIC DUCTILITY

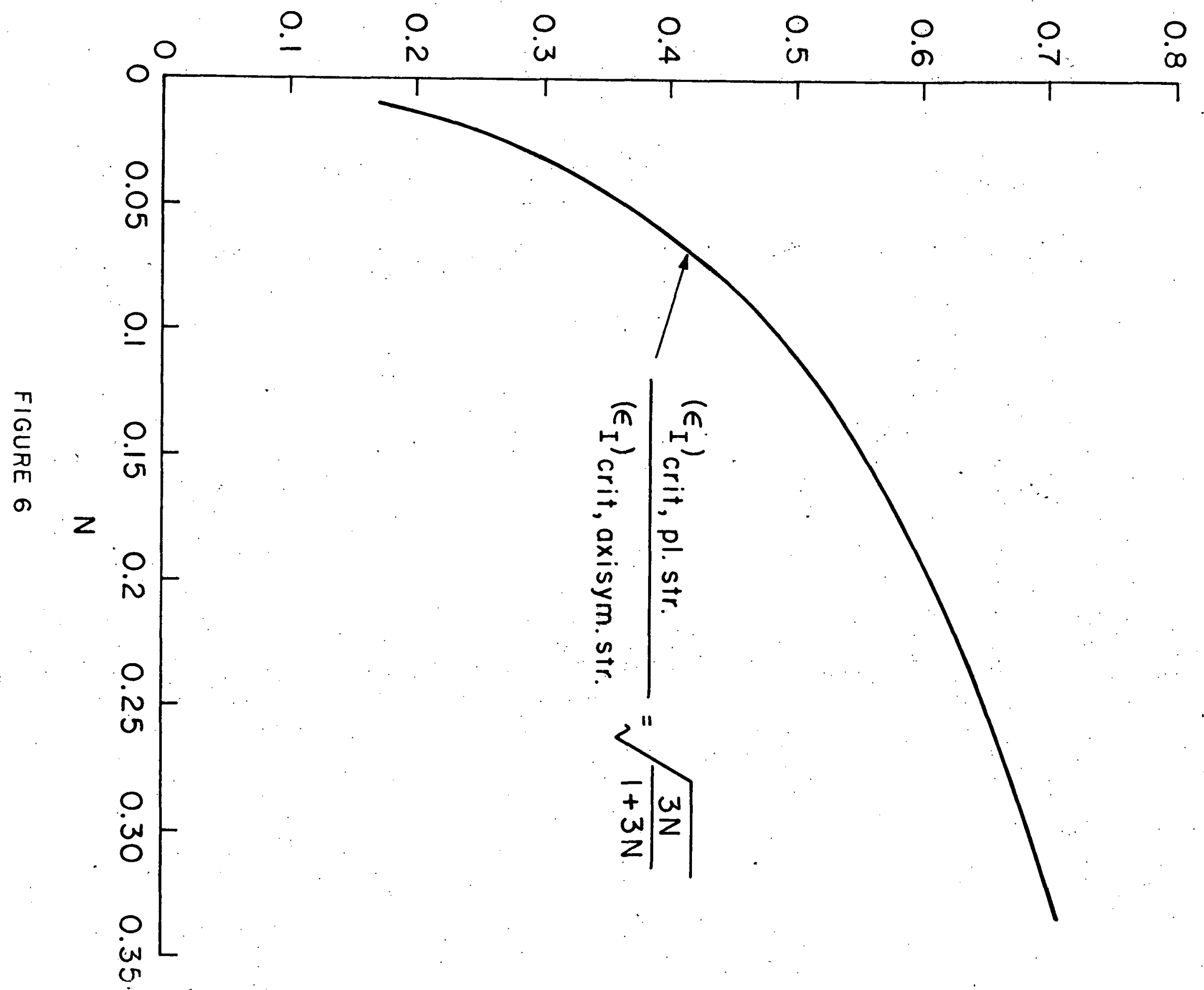




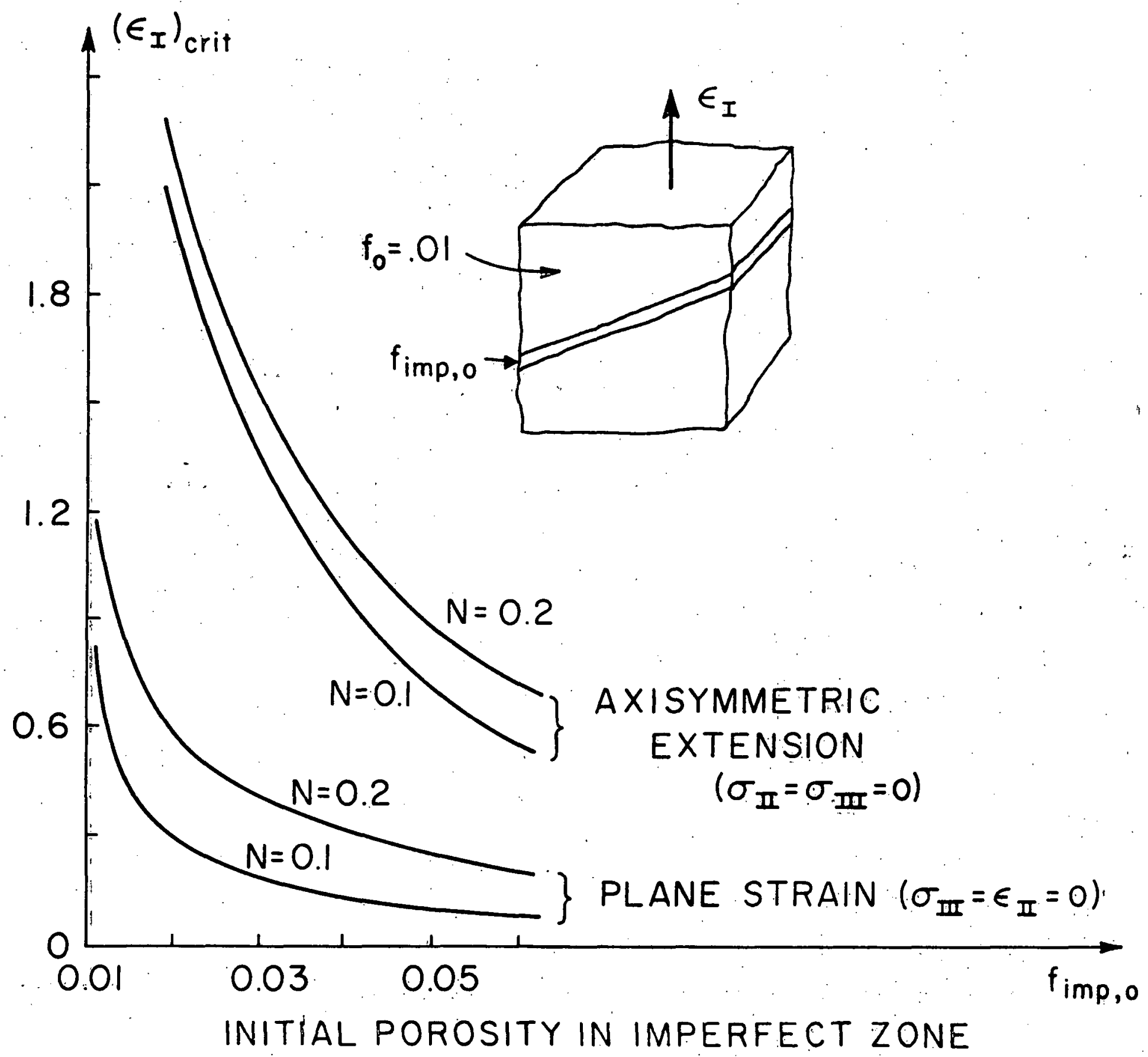

FIGURE 7 


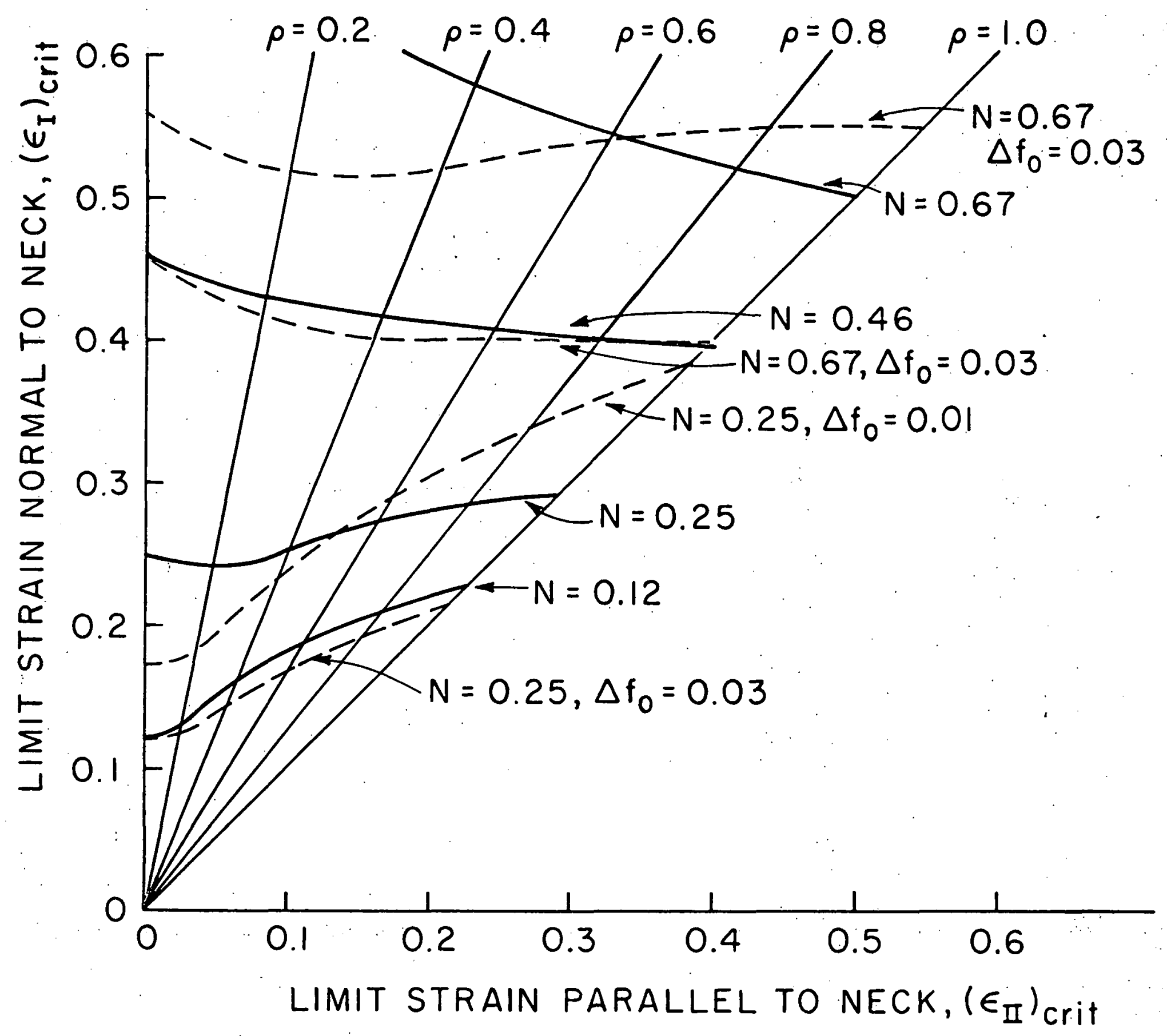

FIGURE 8 


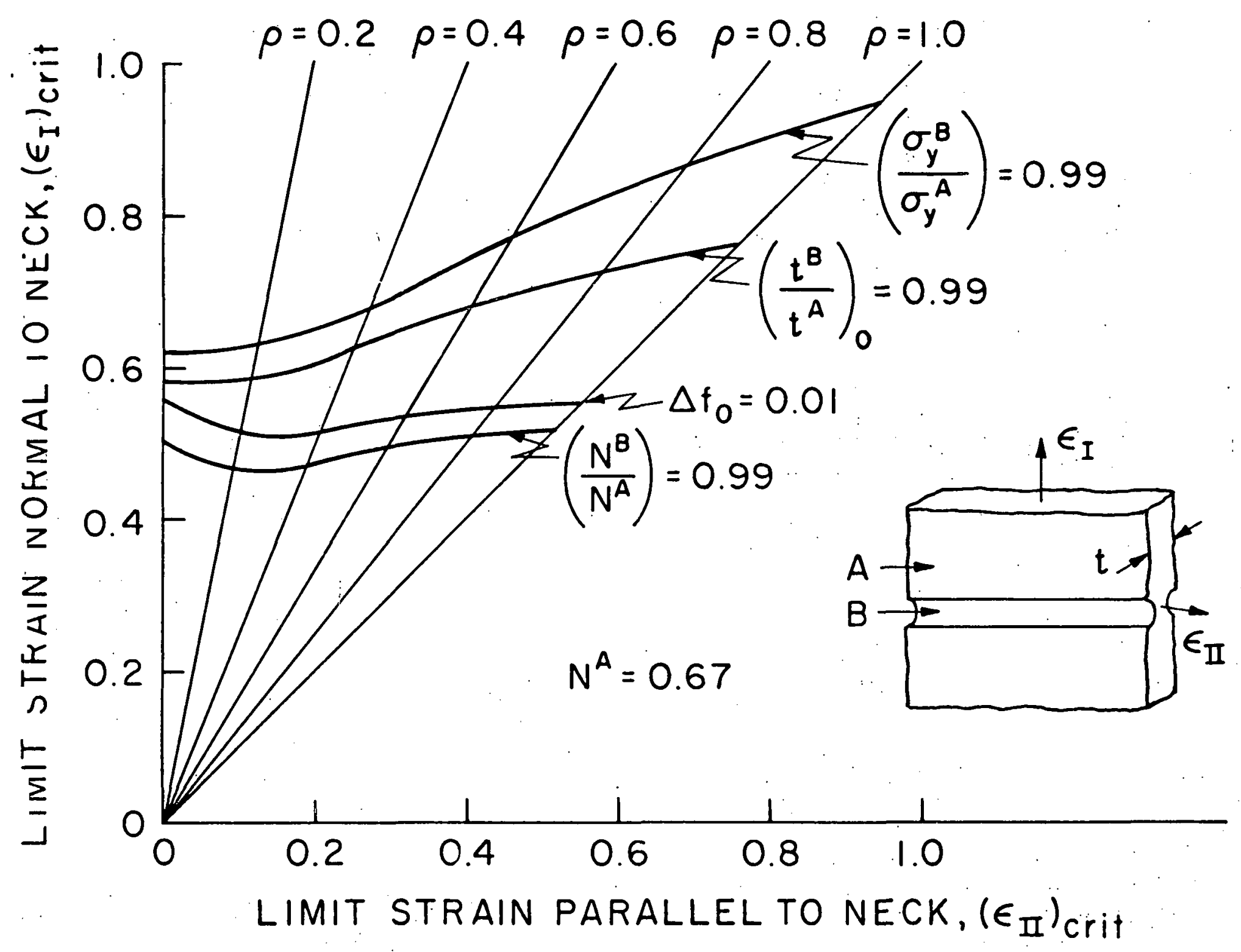

FIGURE 9 